\title{
ANTIPROTON PRODUCTION AND ACCUMULATION
}

\author{
E.J.N. Wilson \\ CERN, Geneva, Switzerland
}

\section{TABLE OF CONTENTS}

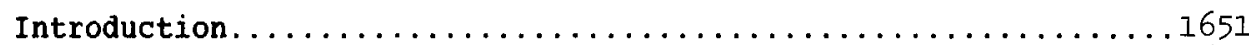

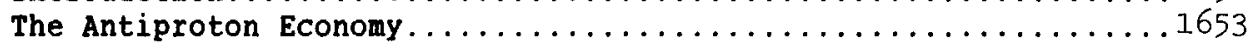

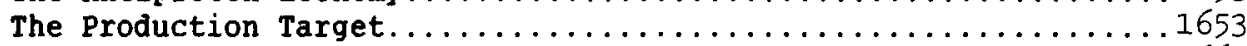

Antiproton Production Cross-Section...................... 1660

Collection of Antiprotons from the Target..................... 1663

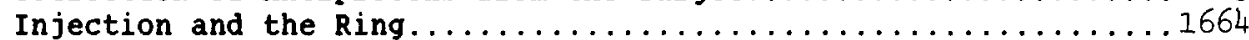

The Life Cycle of Antiprotons in the AA................... 1668

The Cooling Systems.................................. 1673

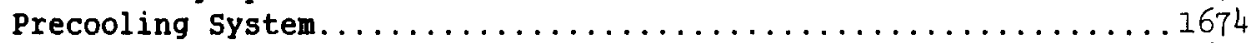

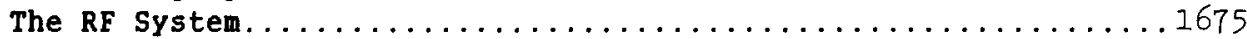

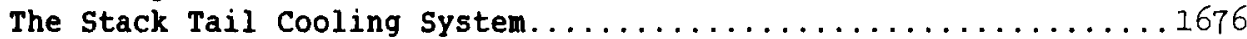

Stack Core Cooling................................... 1677

Performance and Improvements.......................... 1678

References................................... 1680

\section{ABSTRACT}

This paper describes the Antiproton Accumulator storage ring, the heart of the CERN antiproton facility. The principles of the systems which collect, inject, stack, cool, and eject the daily ration of antiprotons are explained. The limitations of the Antiproton Accumulator are discussed, and present plans to improve flux by building an additional collector ring are described. 


\title{
ANTIPROTON PRODUCTION AND ACCUMULATION
}

\author{
E.J.N. Wilson \\ CERN, Geneva, Switzerland
}

\section{INTRODUCTION}

Antiprotons have always been a rare commodity. The threshold energy for producing them by bombarding a fixed target with protons is just over $6 \mathrm{GeV}$, and it was with protons of this energy that the Bevatron produced the first trickle of antiprotons in the 1950's. More powerful accelerators, the AGS and PS, followed. But, even with primary protons of $25 \mathrm{GeV}$ and above available, one thousand protons are needed to produce each antiproton. Only about one in a thousand of those produced falls in the narrow range of angles and momenta which can be collected and transported by a beam line to an experiment. Nevertheless, in the 1960's and '70's many successful experiments were carried out with pulses of 106 antiprotons produced from beams of 1012 or 1013 protons incident upon a fixed metal target. Then came the proposal to collide antiprotons circulating in the SPS with a much more tenuous target, a counter-rotating bunch of protons. This was in order to raise the energy available in the centre of mass to $500 \mathrm{GeV}$ in the hope of producing $W$ and 2 particles. Clearly 106 antiprotons would not suffice to produce observable rates of $W$ and 2 's. It would take at least a day, at the normal 2.4-second repetition rate of the PS, to accelerate enough pulses of $26-\mathrm{GeV}$ protons to make sufficient antiprotons. Each of the 36,000 antiproton bursts would therefore have to be collected in a small storage ring, the Antiproton Accumulator. The best momentum to collect the antiprotons produced by $26-\mathrm{GeV}$ protons is $3.5 \mathrm{GeV} / \mathrm{c}$, and it was for this energy that the storage ring was designed.

To collect as many antiprotons as possible, the AA ring would need a large aperture, but even its large aperture would overflow in a few pulses. Somehow, each injected pulse of antiprotons would have to be progressively compressed into a smaller region of phase space to allow room for more pulses to follow. Fortunately a new technique, stochastic cooling, was available to do just this.

At the time of launching the Antiproton Project there had been barely time to demonstrate that stochastic cooling worked, nevertheless it was decided to go ahead. The machine was constructed in only two years and now runs non-stop for over a month at a time.

The AA can be seen as a small circle one quarter of the diameter of the PS in the layout of CERN shown in Fig. 1. The 26-GeV/C PS accelerates protons in the clockwise sense. Its beam hits a target just before the $A A$ and produces antiprotons. After collection, the 3.5-GeV/c antiprotons are ejected around the reverse loop, TTL2, back to the PS, where they are accelerated to $26 \mathrm{GeV} / \mathrm{c}$ as they circulate in the opposite sense to protons. They then pass down a transport line, TT70, to the SPS. 


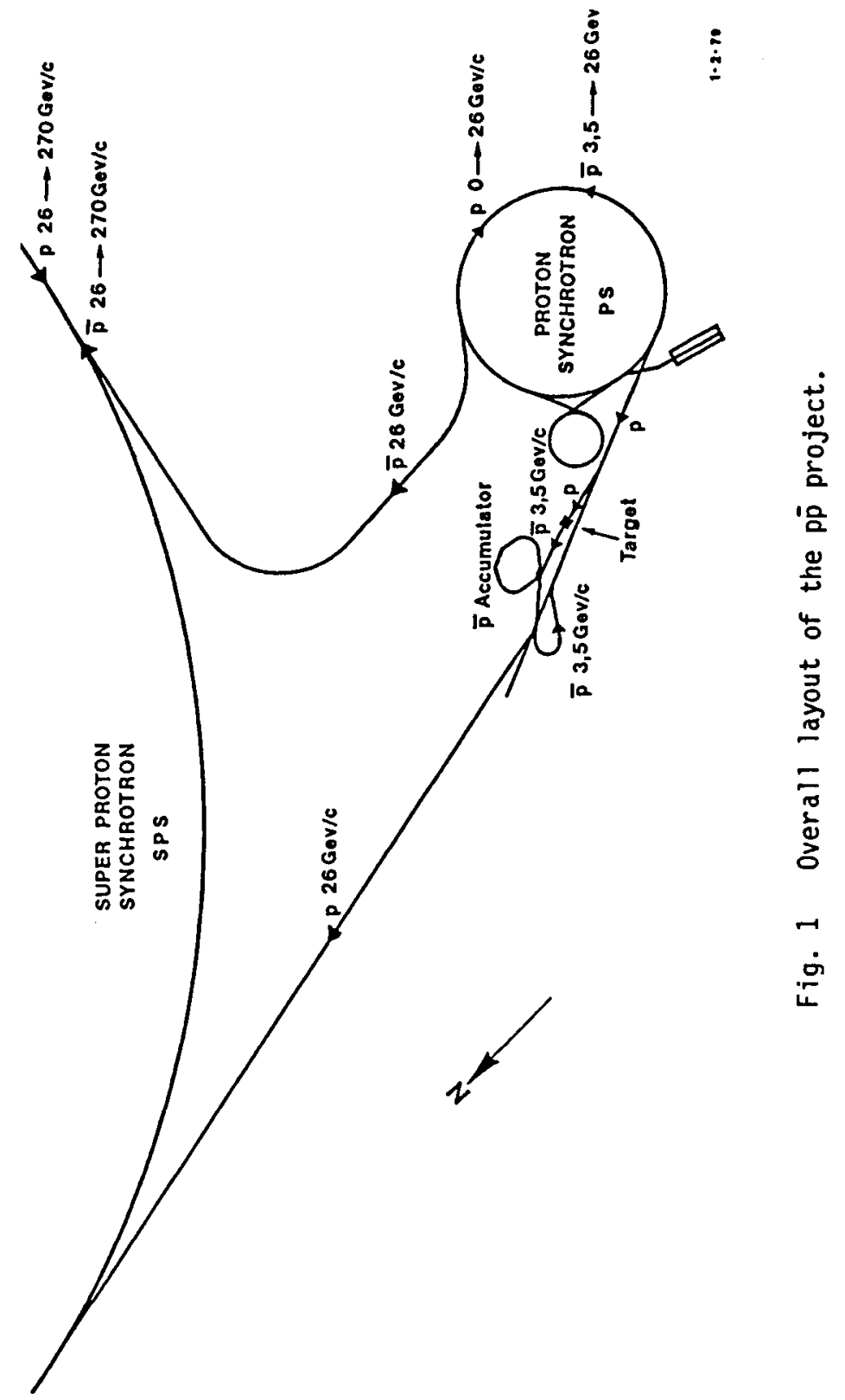


A more detailed layout of the AA is shown in Fig. 2. Here we see the lozenge-shaped ring of the AA. The production target is midway along the injection line entering from the left. Immediately after the target is a pulsed magnetic horn which acts as a powerful collimating lens for the antiprotons. The injected beam joins the ring at twelve o'clock in Fig. 2. The ejected antiprotons leave at the same point through the ejection line, which heads right, out of the picture.

In this talk I shall concentrate on the various systems of the AA which process the antiprotons from the moment they are produced until, after accumulation and cooling, they are ejected towards the PS. Not covered are the process of accelerating the daily ration of antiprotons to $26 \mathrm{GeV} / \mathrm{c}$ in the PS and then to $270 \mathrm{GeV} / \mathrm{c}$ in the SPS1-2 and the technique of producing the intense $26-\mathrm{GeV} / \mathrm{c}$ primary proton beam of the PS so that it is short enough to just fill one turn of the AA with antiprotons.2-3 I shall however describe the principles of stochastic cooling.

\section{THE ANTIPROTON ECONOMY}

The flux of antiprotons is matched to the needs of the SPS. Once a day the 3 antiproton bunches circulating in this machine will have grown transversely to outlive their useful luminosity. They must be refreshed with 3 bunches, each containing 3 . 1010 antiprotons. With losses here and there, this represents a daily demand of 1011 p's or a stacking rate of $5 \times 10^{9}$ /hour.

The PS accelerates upwards of 1013 primary protons per $26-\mathrm{GeV} / \mathrm{c}$ pulse at a repetition rate of 2.4 seconds. The yield of antiprotons injected in the $A A$ is about $6 \times 10^{-7}$ per incident proton. About 508 of these are lost due to precooling, capture, and stack tail inefficiencies and simply because stacking must stop for an hour or so a day to prepare a transfer. The result is a stacking rate which satisfies the present appetite of the Spp̄s. Table 1 summarizes the performance in Spring 1982 - the height of the search.

In addition, the $A A$ ring has run to provide the ISR with antiprotons, and there are also runs in which it feeds LEAR, a low energy accelerating or decelerating and stretching ring for antiprotons, which requires weak antiproton pulses every hour.

The demand on the AA is likely to increase when separators in the SPS are installed to hold the beams apart at unwanted crossings and reduce the beam-beam tune shift, and there is a project afoot to increase this flux by a factor 10 .

\section{THE PRODUCTION TARGET}

The primary proton beam consists of five short bunches of $26-\mathrm{GeV} / \mathrm{c}$ protons which arrive within 470 ns and are focused with pulsed quadrupoles onto the 3-mm-diameter copper rod held in a graphite block. This production target can be found midway along the injection line shown in Fig. 2. A section of the target assembly with cooling fins and a scintillator screen to aid the steering and focusing of the proton beam is shown in Fig. 3 . 


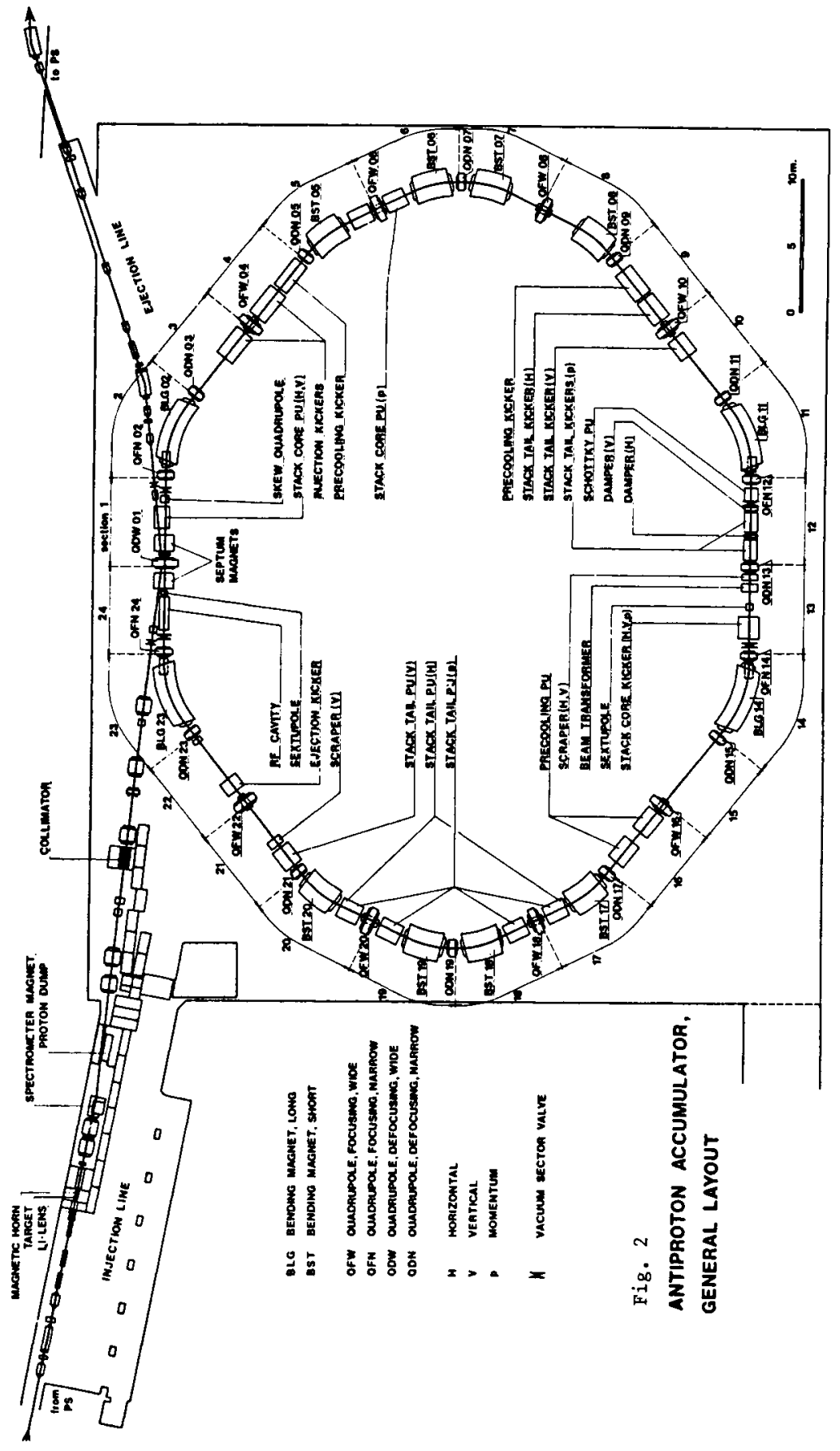




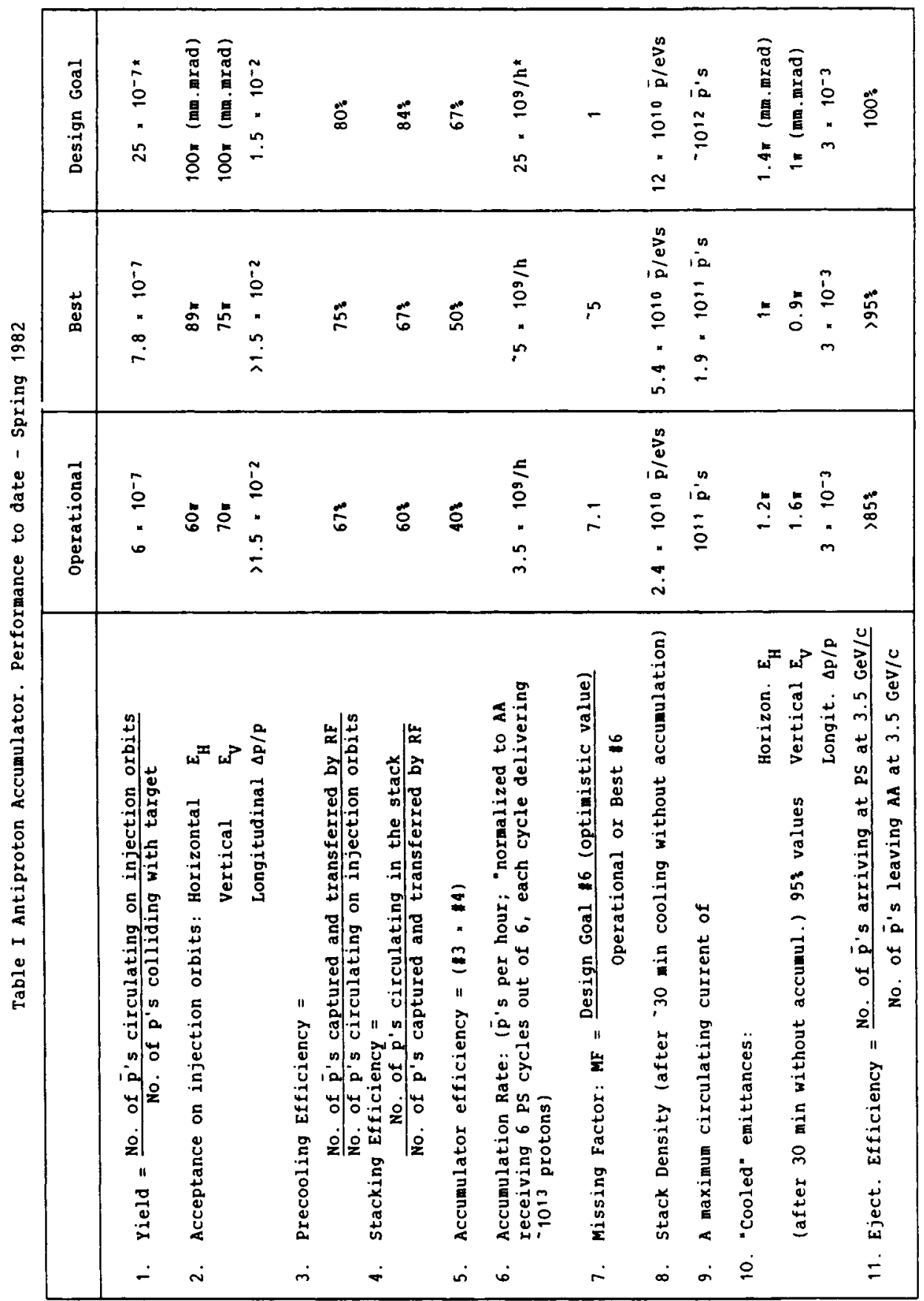


Imagine the target as a source of width $w$ (mm) from which the pulsed horn collection system that follows accepts a cone of particles with semi-angle $\theta$ (mrad) (Fig. 4). The antiprotons transported to the $A A$ from the target lie within an ellipse when projected on one transverse phase plane. The area of this ellipse or emittance is

$\varepsilon=\pi \cdot(w / 2) \theta[\mathrm{m} \cdot \operatorname{mrad}]$.

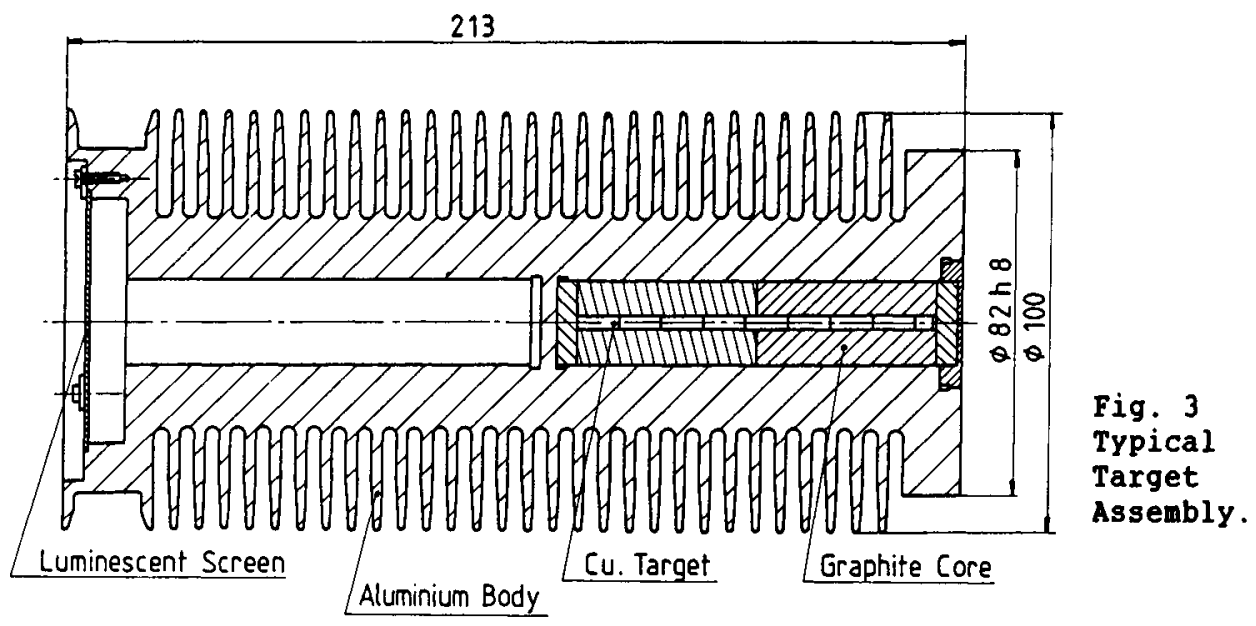

Fig. 4

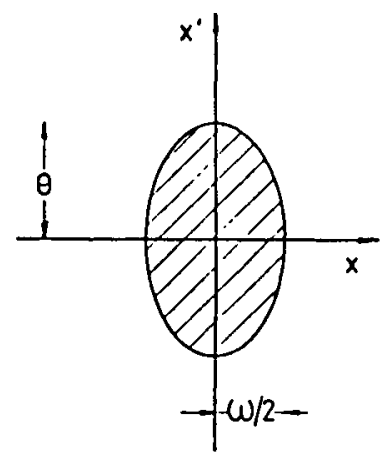

The phase space acceptance, $A$, of the AA which must match this emittance is defined by the elliptical betatron trajectory of the particle that just scrapes the walls of the AA (Fig. 5):

$$
y=\sqrt{A B} \sin (2 \pi Q \theta+\emptyset), \quad y^{\prime}=\sqrt{A / \beta} \cos (2 \pi Q \theta+\emptyset) .
$$

If the maximum displacement, $y$, is limited by the aperture radius of the vacuum pipe, $a$, then the condition that the emittance is accepted is

$$
A=\pi\left(a^{2} / \beta\right)>r(w / 2) \cdot \theta[\operatorname{mm} \cdot \operatorname{mrad}] .
$$




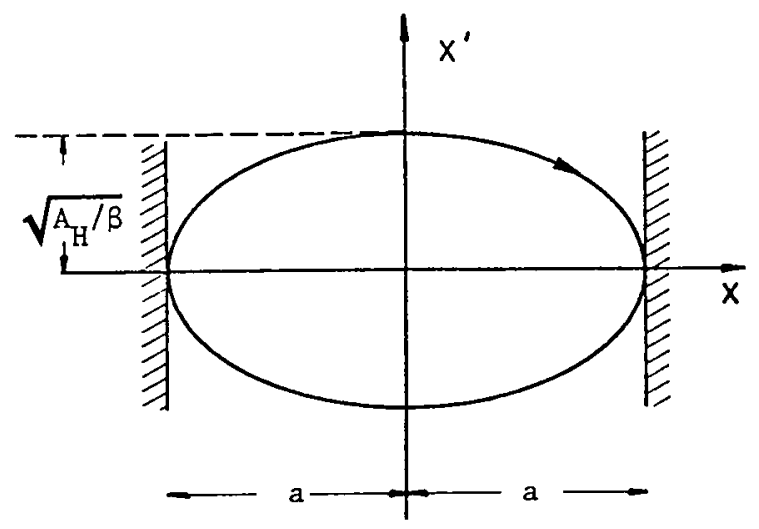

Fig. 5

The AA ring must therefore have sufficient aperture to accept a transverse phase space area which is determined by the product of the target diameter and the angle of the cone of particles transported by the beam line from target to $\mathrm{AA}$. We must try to collect as large a fraction of the production cone as possible, and therefore the target dianeter should be small to keep the AA aperture within the bounds of feasibility. Even with a 2-mm-diameter target the enittance of wanted antiprotons is 100\% mm.mrad in each plane, and some of the AA quadrupole magnets need to have a useful aperture $100 \mathrm{~mm}$ high and $680 \mathrm{~mm}$ wide.

The lower limit to target diameter is set by thermal shock when the primary beam of 1013 to 1.513 " 1013 protons hits it. Figure 6 shows the calculation of temperature fluctuations in a tungsten rod from 6 pulses of protons.5

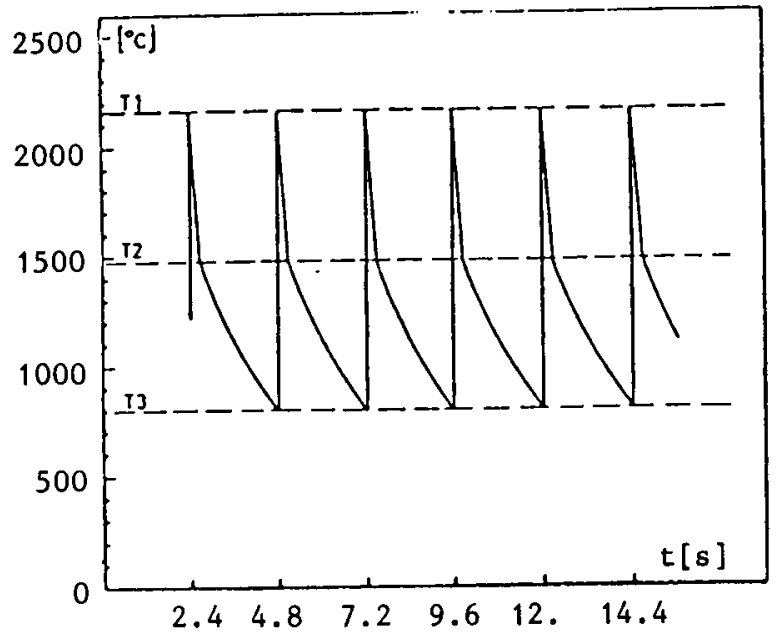

Fig. 6

Since the antiprotons produced at angles of $50 \mathrm{mrad}$ at the beginning of the target will be at a radius of $2.5 \mathrm{~mm}$ by the time they pass the centre of a $10-\mathrm{cm}$-long target, there is a depth of focus 
problem for the particles near the edge of the accepted cone. Figure 7 shows how the emittance diagrams for particles from the upstream (B) and downstream (C) extremities of the target of length $\&$ map on the emittance rectangle from the centre of the target (A) to form a butterfly shape. Instead of an emittance (or machine acceptance) $\varepsilon=$ w日/2 for a target of width $w$ and an acceptance angle of $\theta$, we have

$$
\varepsilon=\frac{\theta(w+2 \theta)}{2} \text {. }
$$
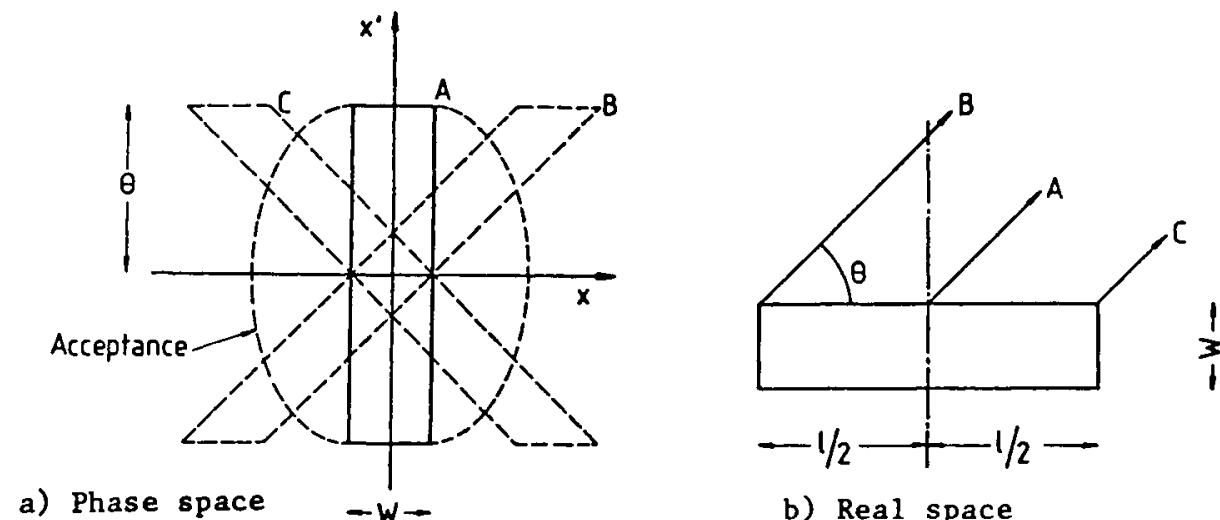

a) Phase space

$-W-$

b) Rea1 space

Fig. 7

Figure 7 also shows how much of this space near the waist of the "insect" contains no antiprotons at all, yet must be included in the elliptical acceptance of the AA ring. Clearly the butterfly shape requires an even larger ellipse than that shown to include all the wings and this too must be paid for in terms of magnet aperture. Of course, this simple treatment takes no account of the distribution of antiprotons as a function of production angle or of the scattering in the membrane of the horn-lens which follows the target. A Monte Carlo simulation is needed to design the real thing.

It is nevertheless apparent that, once target width is fixed by the thermal shock limit, it does not pay to make $l \theta$ larger than $w$. This restricts the choice of target materials to those with interaction lengths that are comparable to $w / \theta(10 \mathrm{~cm}$ for the $A A)$ and rules out light materials like beryllium which otherwise might be preferred for their higher antiproton production cross-section per nuclear interaction.

Early design calculations suggested tungsten would be a good target material because its short interaction length would place the antiproton source in the first few centimetres of the leading face of the target. The pulsed horn-lens can be designed to collect efficiently from this region. Later, measurements showed copper to be marginally better by about $10 \%$. Revised Monte Carlo predictions based on better calculations of scattering and reabsorption in target and horn, and which allow for more recent data on production cross- 
sections, confirm that copper is the best compromise. Although the melting point of copper is lower than that of tungsten, the temperature rise predicted is proportionally smaller.

The depth of focus problem would become even more acute, were we to attempt to collect an even larger solid angle from the target. One way round this is to pass a current down the length of the target. Since the AA was built, such a "conducting target" has been assembled and tested. In this device a pulse of several hundred $k A$ is passed along the target. The pulse is slow enough for the skin depth to be larger than the target radius. The primary beam arrives at the peak of the pulse. Figure 8 shows the principle of the device. The magnetic field that circulates within the conductor is concentric with the axis. Its radial dependence is obtained by considering a circular contour of radius $r$ within the target. If $I$ is the current within this contour, then

$$
\begin{gathered}
\int B \cdot d l=\mu_{0} I, \\
2 \pi \quad r B=\mu_{0} \| J_{0} r^{2}, \\
B=\left[\frac{\mu_{0} J}{2}\right] \mathrm{r} .
\end{gathered}
$$

The quantity in brackets is the gradient of a linear focusing field.

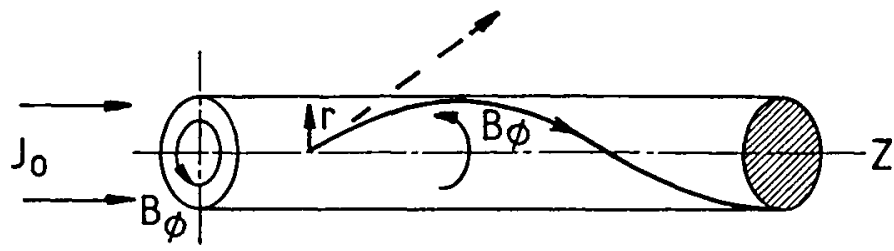

Fig. 8 angle

Within the target the trajectory of an antiproton diverging with

$$
\tan \psi=d x / d s
$$

will be deternined by the equation of motion:

$$
\frac{d(\sin Y)}{d s}=-\frac{e}{\bar{P}} B=-\frac{e_{0} J_{0}}{2 P} \cdot x .
$$

If $\psi \ll \pi / 2$, the solution is

$$
x=x_{0}+x_{0}^{\prime} \sin \sqrt{k s}, \quad x^{\prime}=x_{0} \cos \sqrt{k s},
$$

i.e. equivalent to a lens of strength

$$
\mathrm{k}=\frac{1}{2} \frac{\text { eNo }}{\mathrm{P}} \mathrm{J} \text {. }
$$


The effect of the conducting target is to transmit all antiprotons created in its length so that they emerge from a disc at the end of the target, rather like a light guide. The butterfly can be made to fold its wings into an elliptical shape which is better matched to the elliptical acceptance diagram of the machine. There is a critical strength per unit length which just removes the depth of focus problem of a normal target. It is given by

$$
k=\frac{1}{2} \frac{e}{p} \mu_{0} J=\left(\frac{\pi}{l}\right)^{2}
$$

where $l=$ target length.

with this strength the beta value to which the subsequent transport system is to be matched is just $\beta=1 / \sqrt{k}$.

The gain from the conducting target for the AA situation is predicted to be about $40 \%$, and this is confirmed by tests.

\section{ANTIPROTON PRODUCTION CROSS-SECTION}

Information on antiproton production cross-sections was rather sparse at the time the AA was proposed. The last survey of the differential production cross-section as a function of production angle, of primary and secondary momentum, and for different target materials had been made at the PS in 1962.6 with the benefit of hindsight, many months of AA running, and prediction based on fitting more recent measurements, we now know that the particular data point ( $\mathrm{Pb}, 0^{\circ}$, and $3.5 \mathrm{GeV} / \mathrm{c}$ ) taken for the AA design was a factor 1.7 too high. It was in fact right at the lowest end of the survey, where rejection against background was worst. This is indicated by the error bars in Ref. 6 .

In designing new facilities the reader is referred to Hojvat and van Ginneken, 7 who have fitted the following empirical expression to world data. The left-hand side is the invarient differential production cross-section in the centre of mass.

$$
\begin{gathered}
W=2\left(\frac{E}{\sigma_{a b s}}\right) \frac{d^{3} g}{d p^{3}}=2\left[k\left(1-x_{R}\right)^{m} \exp \left(-3 p_{t}^{2}\right)\right] \times\left[1-24 s^{-2} \exp \left(8 x_{R}\right)\right] \\
*\left[a \exp \left(b p_{t}^{2}\right) \exp \left(-c x_{R}\right)\right]
\end{gathered}
$$

where $\quad x_{R}=E / E_{0}=\bar{p}$ energy/largest allowed,

$\sigma_{\text {abs }}=$ absorption cross-section,

$k=0.065$
$m=8.0$ for $\mathrm{p}$ and $\mathrm{n}$ projectiles,

$a=1.7$

$\mathrm{b}=1.4$

$c=2.8$

for $\mathrm{Cu}, \mathrm{w}$, and $\mathrm{Pb}$.

The three factors are claimed to be:

i) a fit to data in the high energy scaling region, 
ii) a term for scaling at lower energy,

iii) nuclear mass dependence.

This invariant cross-section may be converted into the more familiar differential cross-section in the laboratory which is normalized to each interacting proton:

$$
W=\frac{2 E}{p^{2}}\left(\frac{d^{2} N}{d W d p}\right)_{1 a b}=\left(\frac{2 E}{n_{a b s}}\right)\left(\frac{d^{3} a}{d p^{3}}\right)_{c o m} .
$$

Figure 9 (from Ref. 8) shows how this theoretical fit compares with world data. The data point taken for AA design calculations is clearly above present-day predictions. Figure 10 shows how we expect W to vary as a function of antiproton momentum in the lab compared with data for production by $24-\mathrm{GeV} / \mathrm{c}$ protons.
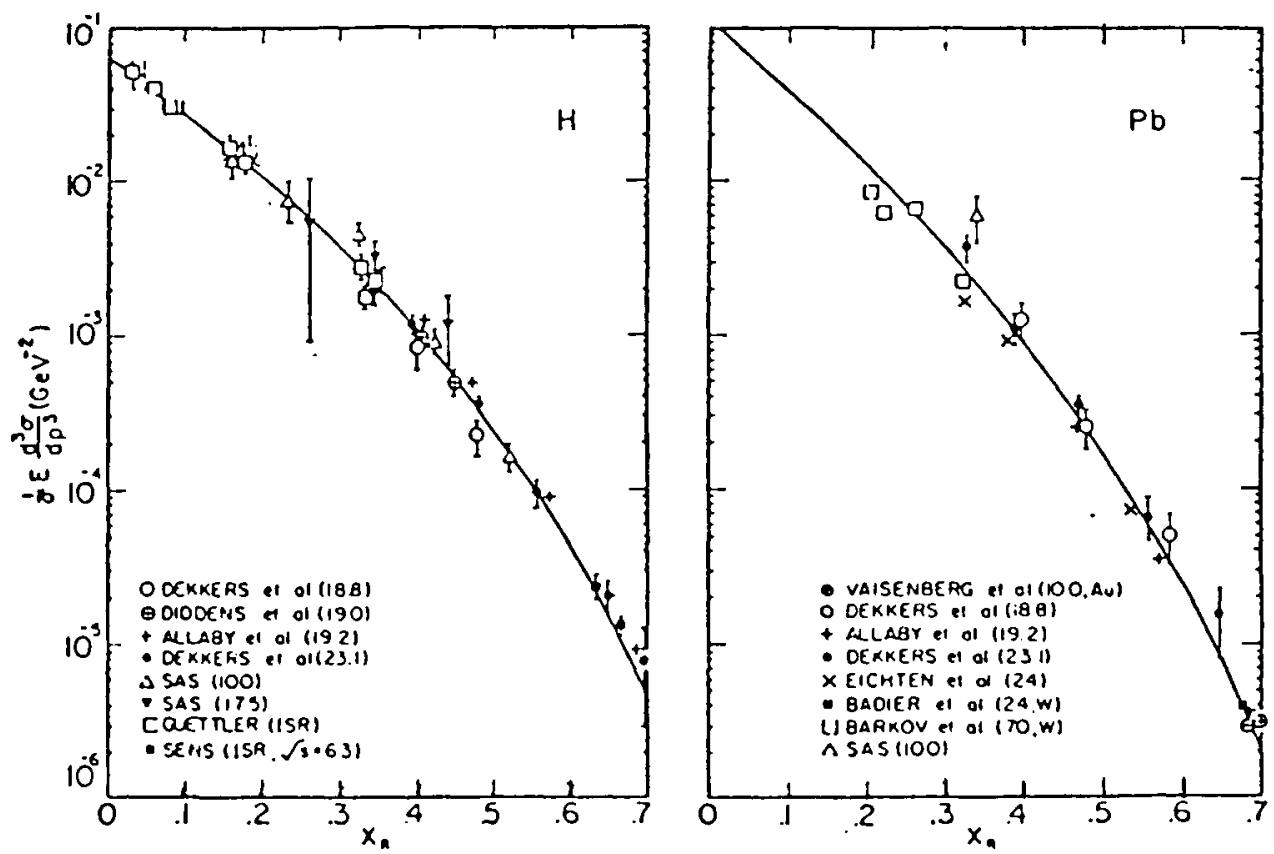

lavariant differencial $\bar{p}$ production cross soction vs. $x_{n}$ with $p_{1}-0$ held constant and $x \rightarrow \infty$.

Fig. 9

Figures 10 and 11 (also from Ref. 8) show how the differential cross-section per nucleon falls with increasing atomic mass, and we can get some idea of the dimensions of the production cone from the fact that the cross-section is a factor of 2 lower at $100 \mathrm{mrad}$ than at zero degrees. Since the AA collects a cone of about 50 mrad halfangle,there would still be a gain to be had if we could double the acceptance. The first element that limits the acceptance angle, the magnetic horn, is discussed in the next section. 


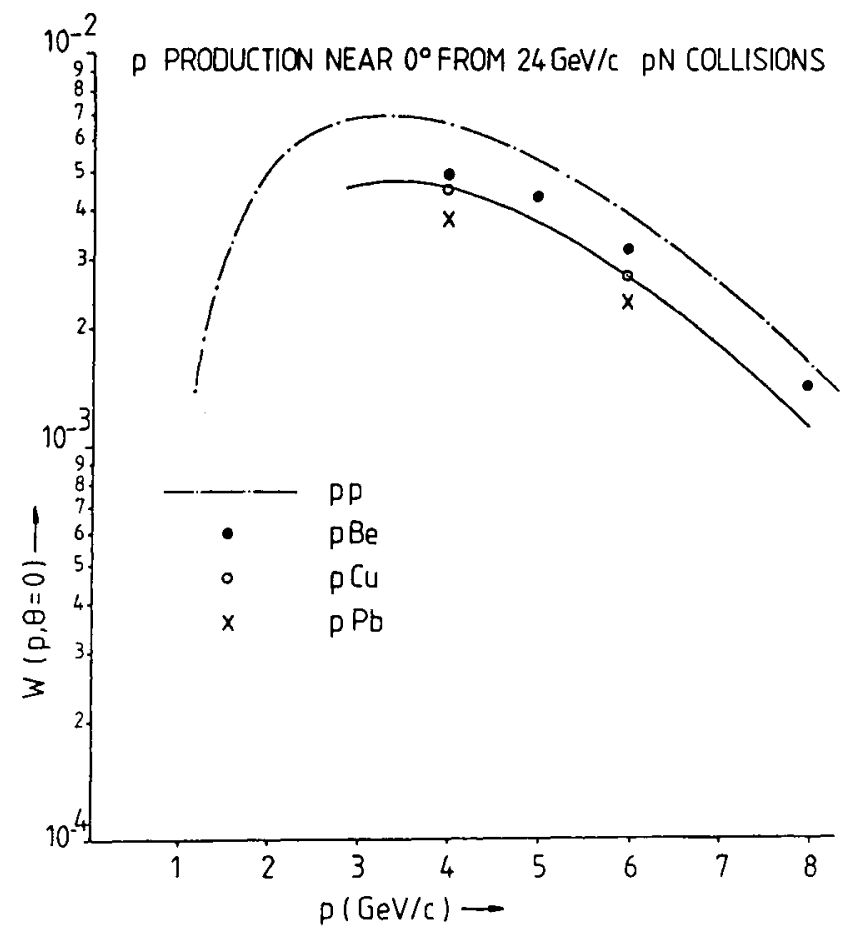

Fig. 10

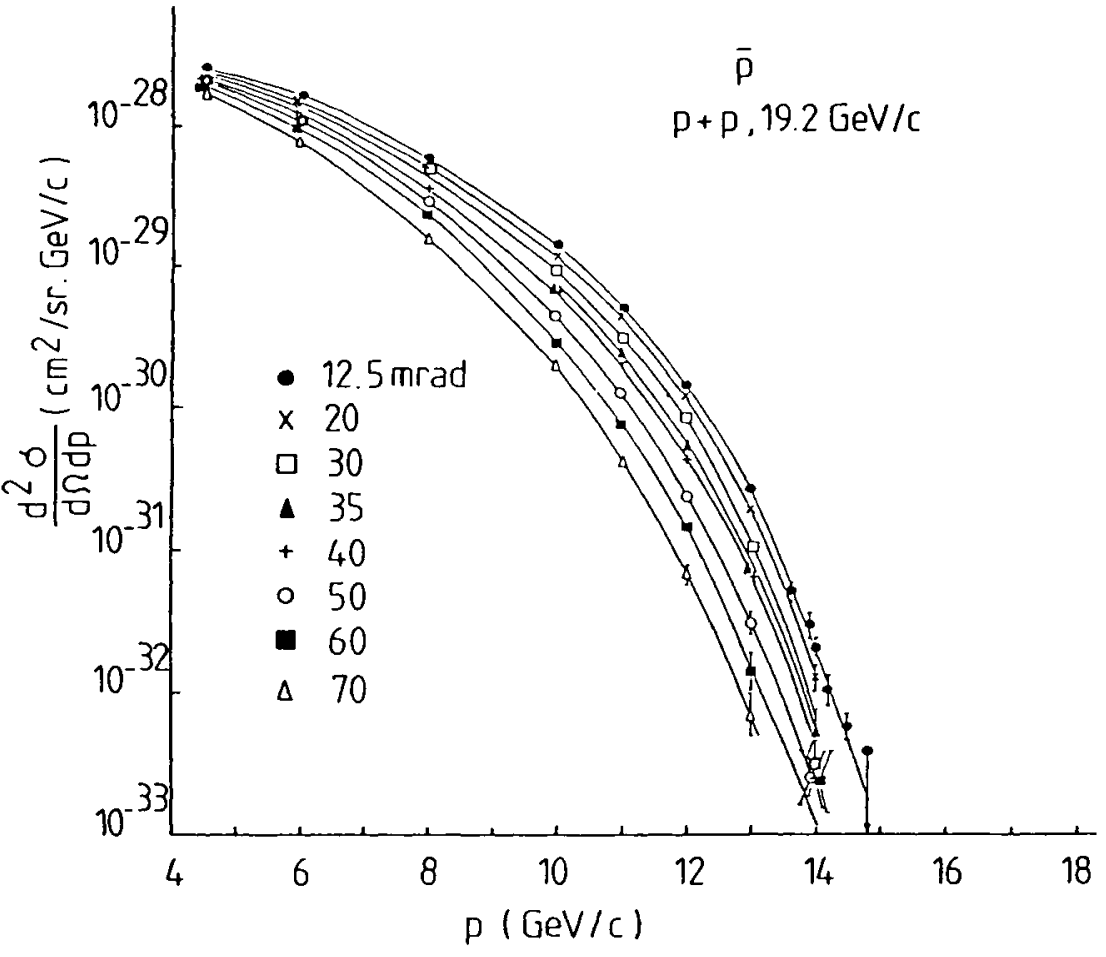

Fig. 11 


\section{COLLECTION OF ANTIPROTONS FROM THE TARGET}

The focusing system used to collect secondary particles from a target usually consists of a pair of quadrupoles of opposite polarity. We soon found such a system was not able to collect a large enough solid angle. The angle was large in one plane where the polarity of the quadrupoles was FD, but in the DF plane such a doublet has a long focal length and small acceptance. The system also suffered from chromatic aberration which prevented a good match being achieved between target and storage ring for off-momentum particles.

The device finally chosen to focus antiprotons diverging from the target was a horn-shaped conductor similar to that used to focus the parent particles in neutrino beams. It is the inner conductor which does the job of focusing, the outer cylinder (Fig. 12a) merely carries the return current. The horn is cylindrically symmetric about the axis of the target. There is no field within the inner conductor, but outside the horn shape a magnetic field circulates about the beam axis and has a radial dependence:

$$
B_{\emptyset}=\frac{\mu_{0} I}{2 \pi} \frac{1}{r}
$$

where $r$ is the radial coordinate, and $I$ is the total current.

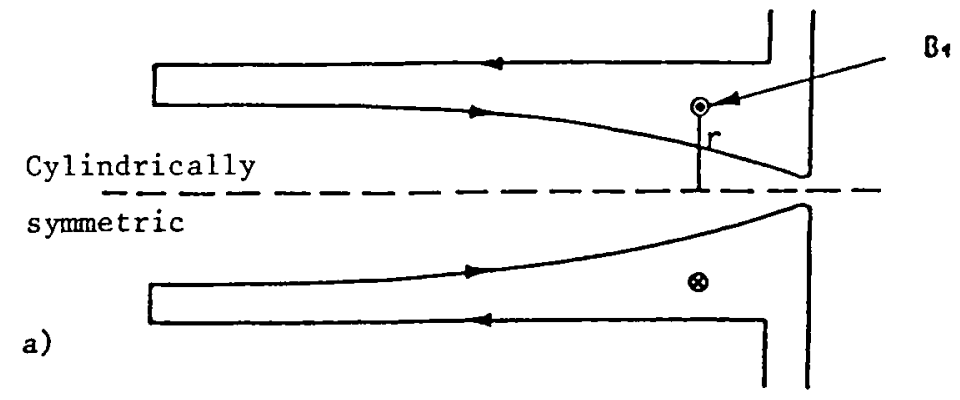

b)

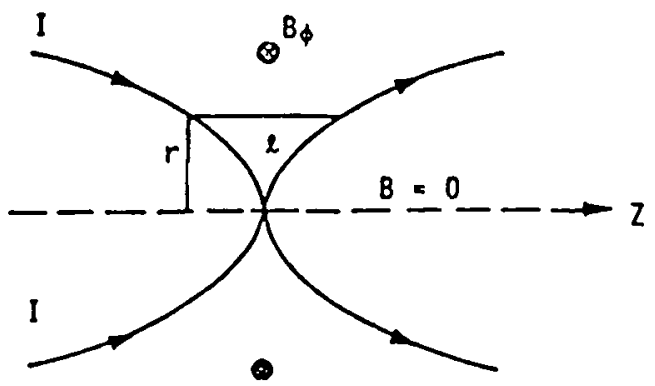

Fig. 12

Imagine a particle diverging from the target at an angle $\theta$. Its radial position as it crosses the current sheet into the magnetic field region is $r$. The field it encounters there will be inversely proportional to $r$. Since the horn is parabolic, the path length in the field $a r^{2}$ and the deflection it receives, 


$$
\theta=\frac{1}{B_{\theta}} \int B_{\emptyset} \mathrm{ds},
$$

will be proportional to $r$, as in a linear focusing lens. An appropriate choice of current ensures that this deflection just cancels the initial divergence, 8 . of course, the calculation is complicated by the fact that $r$ is not simply proportional to $\theta$, but this is taken into account in the program used to find the best shape and location for the horn.

Early designs from Novosibirsk showed a double parabola (Fig. $12 b)$ but calculations allowing for scattering in the horn showed the present geometry to be better. Scattering is more critical at the downstream end. 9

The horn current in the $\mathrm{AA}$ is about $160 \mathrm{kA}$ and in its design the mechanical shock of repeated pulsing must be balanced against the scattering effect in arriving at an optimum thickness. The extra thermal shock of a defocused proton beam accidentally striking the horn in synchronism with the current must be avoided. Figure 13 shows the practical design of the horn used in the AA.

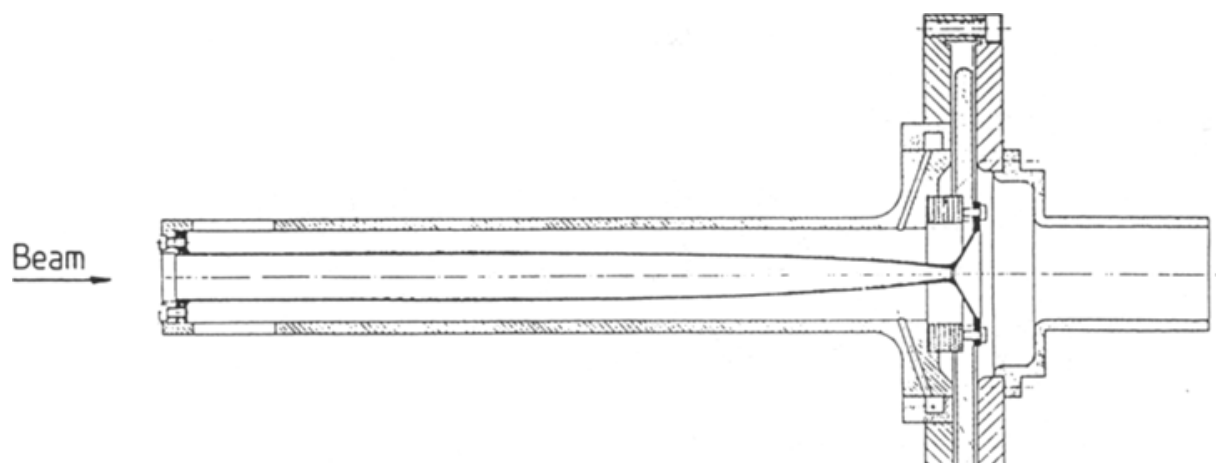

Fig. 13

In more recent times an alternative to the horn has emerged (from the same stable, Novosibirsk). This device, the lithium lens, consists of a current-carrying bar of lithium. The field in the bar is like that of the conducting target and to a first approximation has the effect of a simple symmetric lens. Lithium is chosen because of its favourable ratio of conductivity to scattering. Figure 14 shows the cross-section of such a device developed at FNAL and tested in the AA production beam for more than 106 pulses.

\section{INJECTION AND THE RING}

Compared with the high technology close to the target, the rest of the $A A$ injection line is very conventional. A single bending magnet deflects $3.5-\mathrm{GeV} / \mathrm{c}$ antiprotons away from the spent beam of $26-\mathrm{GeV} / \mathrm{c}$ protons, which plough into a dump. The transport line must be matched to the phase space at the exit of the horn and to the acceptance of the AA. When, as part of our improvement programme, 


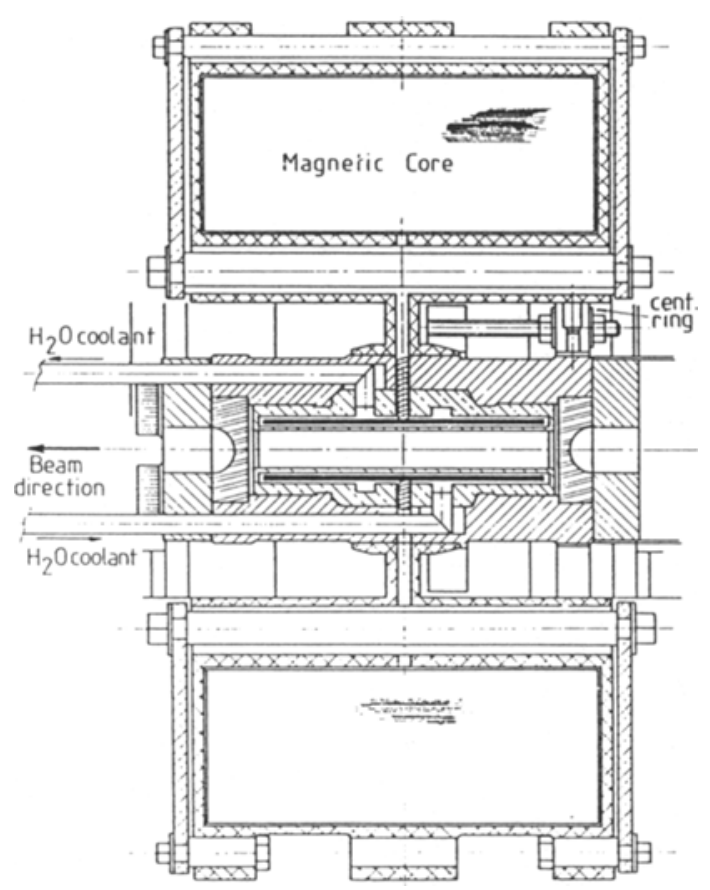

Fig. 14 .

conducting targets and a lithium lens are used to collect a higher flux, we will install a double spectrometer configuration to reduce the momentum bite of unwanted hadrons which accompany the antiprotons into the AA ring building. Ideally this bite, defined by a collimator in the wall between target area and $A A$, should be no bigger than the momentum spread the AA can swallow. However, at present, with a single, rather weak bend and a hole large enough to accept the full transverse phase space, about 6 times the wanted momentum bite passes through.

Figure 2 indicates the layout of the AA ring, whose structure is a FODO lattice of 12 periods. It has two long straight sections, at 6 $0^{\prime}$ clock and $120^{\prime}$ clock in the figure, where the dispersion function $D$ is zero and the beam is only $30 \mathrm{~mm}$ in radius. The lattice is designed to have a dispersion that rises to its full value in the first three half-periods of the FODO structure and is maintained around the arcs at $30^{\prime}$ clock and $90^{\prime}$ clock, where the position-sensitive pick-ups for the various cooling systems are placed. The matching of the dispersion function and its average value, which influences transition energy and hence the rate of stochastic mixing, was adjusted in designing the lattice by adjusting the strength of the four long bending magnets at the ends of the long straight section relative to the eight short magnets at $30^{\prime}$ clock and $90^{\prime}$ clock.10 A trim power supply on one set of magnets allows a final empirical adjustment in setting up to ensure the orbits are centred in the long straights. The $60^{\prime}$ clock long straight section is filled with cooling kickers which reduce the momentum spread of the stack. Any correction, $\delta p$, which is made to the momentum of a particle by cooling should ideally 
be applied where $D=0$ or the particle will suddenly start oscillating about a new closed orbit displaced by $D \delta p / p$ and corresponding to its new momentum. Even if it had no betatron amplitude before the kick, it would acquire an amplitude D $8 p / p$ (Fig. 15).

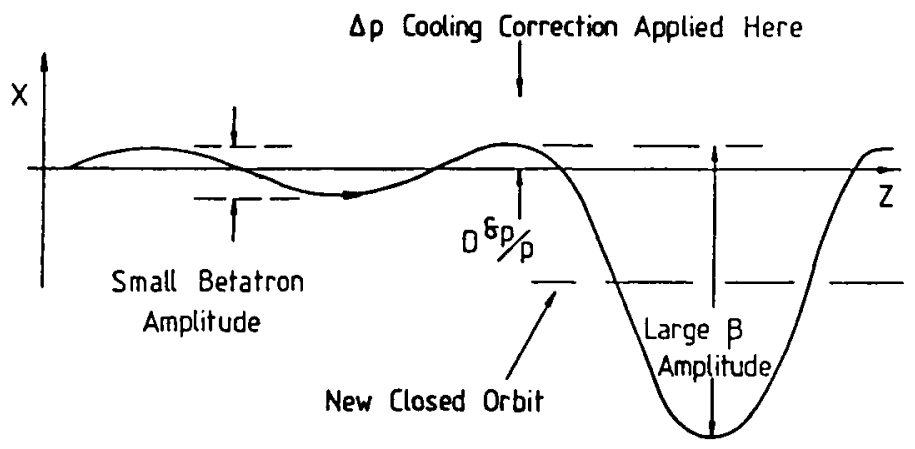

Fig. 15

The long straight section at $120^{\circ}$ clock is used mainly for injection and ejection trajectories. In a classical single-turn injection scheme the injected beam approaches the vacuum chambers of the circulating beam at an angle and then is bent to ride alongside the chamber, precisely parallel to the circulating orbit but displaced from it (Fig. 16). The magnet that does the bending has a coil in the form of a thin septum or current sheet since it is this coil which separates the incoming and circulating beams, isolating the circulating particles from the magnet's field. The septum must be thin to allow the beams to come close together. In the AA the septum magnet is in two halves symmetrically placed about the lattice quadrupole at $60^{\circ}$ clock (Fig. 17).

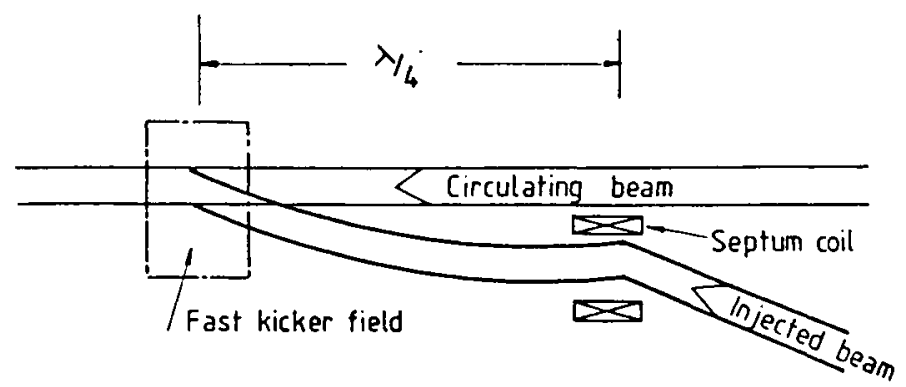

Fig. 16

After $90^{\circ}$ of betatron phase advance the relative displacement of the two beams is converted into an angle and because the two beams were initially parallel their physical displacement is now zero. A fast kicker magnet placed at this azimuth is fired just before the incoming beam arrives and corrects the angle between the incoming beam and its desired circulating orbit. It must switch off again before the head of the injected beam reaches it again after one turn. 


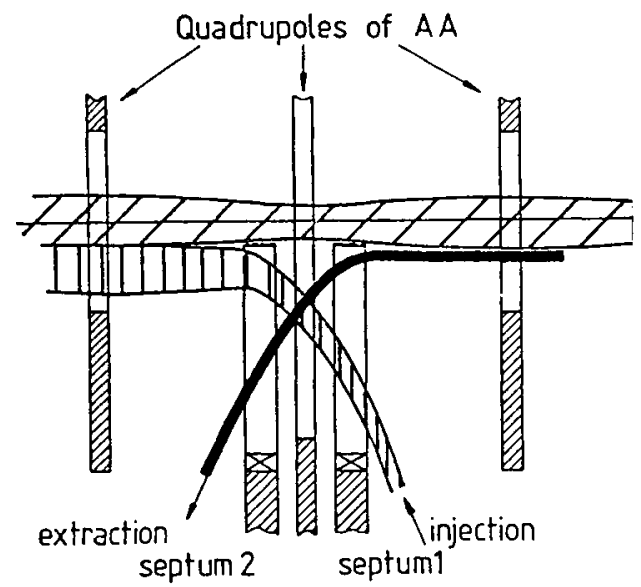

Fig. 17

In the $A A$, ejection is just the mirror image of injection, and both injected and ejected beams use the same septum (see again Fig. 17). The septum is best placed where there is no dispersion so that the displacement of the incoming beam which determines the injection kick is momentum independent. However, the injection and ejection kickers, $90^{\circ}$ in phase from the septum, must be where dispersion is large to ensure that the injected beam, which is in the gap of the kickers, is separated from the stack, which differs by 38 in momentum from the injected particles. The kick must not disturb the stack (Fig. 18).

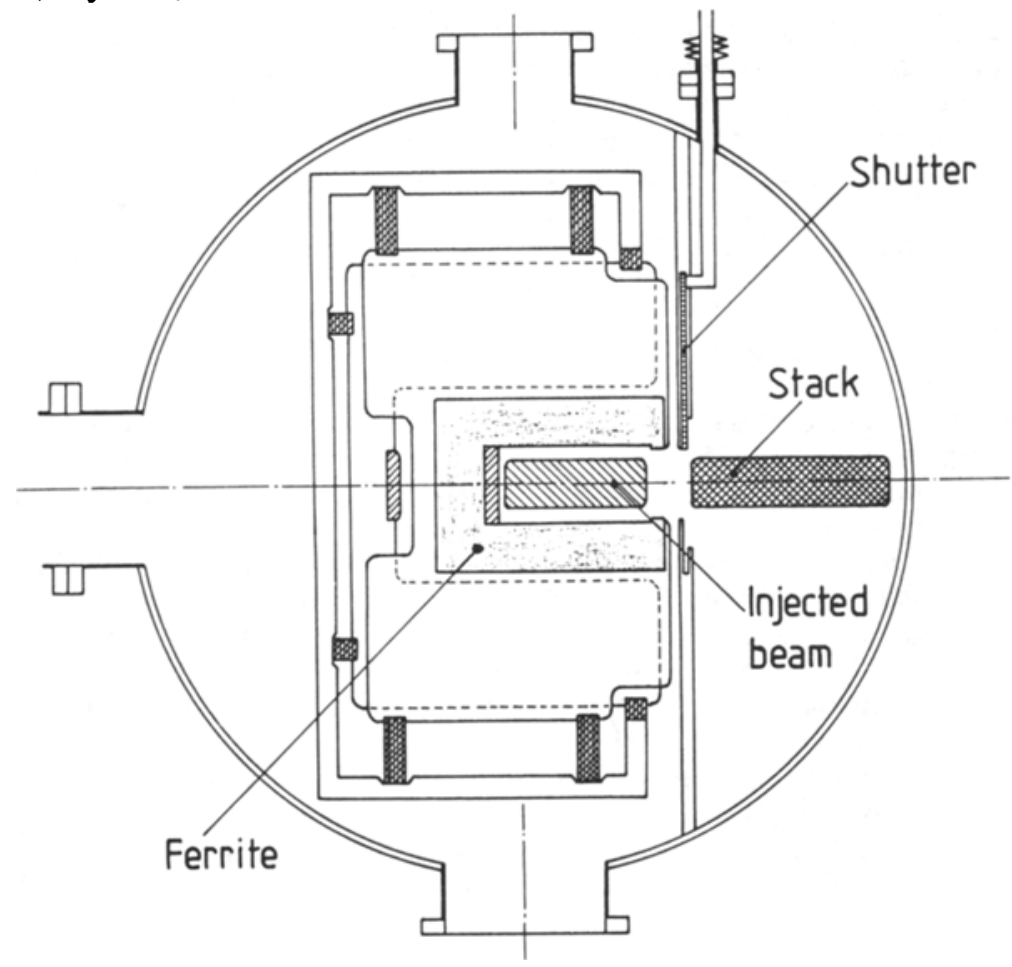

Fig. 18 
So sensitive is the stack to the fringe field of the injection kicker that an eddy current shield or shutter must be raised at the moment of injection to close the open gap of the kicker. Figure 18 shows the arrangement. The moving shutter mechanism, actuated through bellows in a huge bakeable vacuum tank, was one of the most challenging engineering problems in AA construction.

The $Q$ of the machine was chosen to avoid non-linear sum resonances. During running-in it had to be changed slightly to avoid all resonances up to 15 th order. Electrical shunts on quadrupoles were set to rematch the dispersion to zero at this new $Q$ value. The $Q$ variations across the aperture from injection orbit to stack have been adjusted11 by shimming quadrupole ends. The circle of confusion in the $Q$ diagram is only of diameter 0.01 (Fig. 19).

The variation of dispersion between $120^{\prime}$ clock and 3 o'clock, coupled with the large transverse emittances and momentum difference between injected orbit and stack, leads to a much larger vacuum chamber in the arcs. The horizontally focusing quadrupoles in the arcs have an aspect ratio of horizontal to vertical aperture of about $7: 1$ and have greatly extended poles in the horizontal direction (Eig. 20).11 Their nominal horizontal aperture, $68 \mathrm{~cm}$, is twice their magnetic length. They had to be shimmed very carefully to avoid three-dimensional end effects (Fig. 21). New lattice programs were needed to take into account the end field effects on linear focusing and dispersion.

Closed orbits are measured at all F quadrupoles 12 and corrected to within $1 \mathrm{~mm}$ of the centre of the ideal machine by moving selected quadrupoles on their supports. In the vertical plane this adjustment is motorized to allow exploration beam bumps to be made.

Diagnostic devices needed to align the lattice include a precise $Q$ measurement system using betatron side band fed into a spectrum analyzer. There is a beam-scraping system and sophisticated diagnostics to observe injected electrons and to correct their trajectories. It is assumed that antiprotons and electrons behave similarly. A beam current transformer covers the range 107 to $1012 \bar{p}^{\prime} \mathrm{s}$ in circulating beam.

\section{THE LIFE CYCLE OF ANTIPROTONS IN THE AA}

The AA is really two machines in one. The injected beam with as large an emittance and momentum spread as possible rides in the outer half of the vacuum chamber. On the inside of the vacuum chamber, circulating at a different momentum and radial position, is the cooled stack of antiprotons that has been accumulated over many hours of regular pulsing. The life cycle of antiprotons is shown in Fig. 22 .

Liouville's theorem prevents us superimposing the injected particles directly on the stack. In practice an attempt to do so with the kicker which inflects the new pulse onto its orbit would eject the stack. It is not even possible to inject alongside the stack because shutters are needed to prevent the injection kicker's fringe field disturbing the stack.

There are three steps in bridging the gap between injection orbit and stack. They are precooling, RF deceleration, and stack tail 

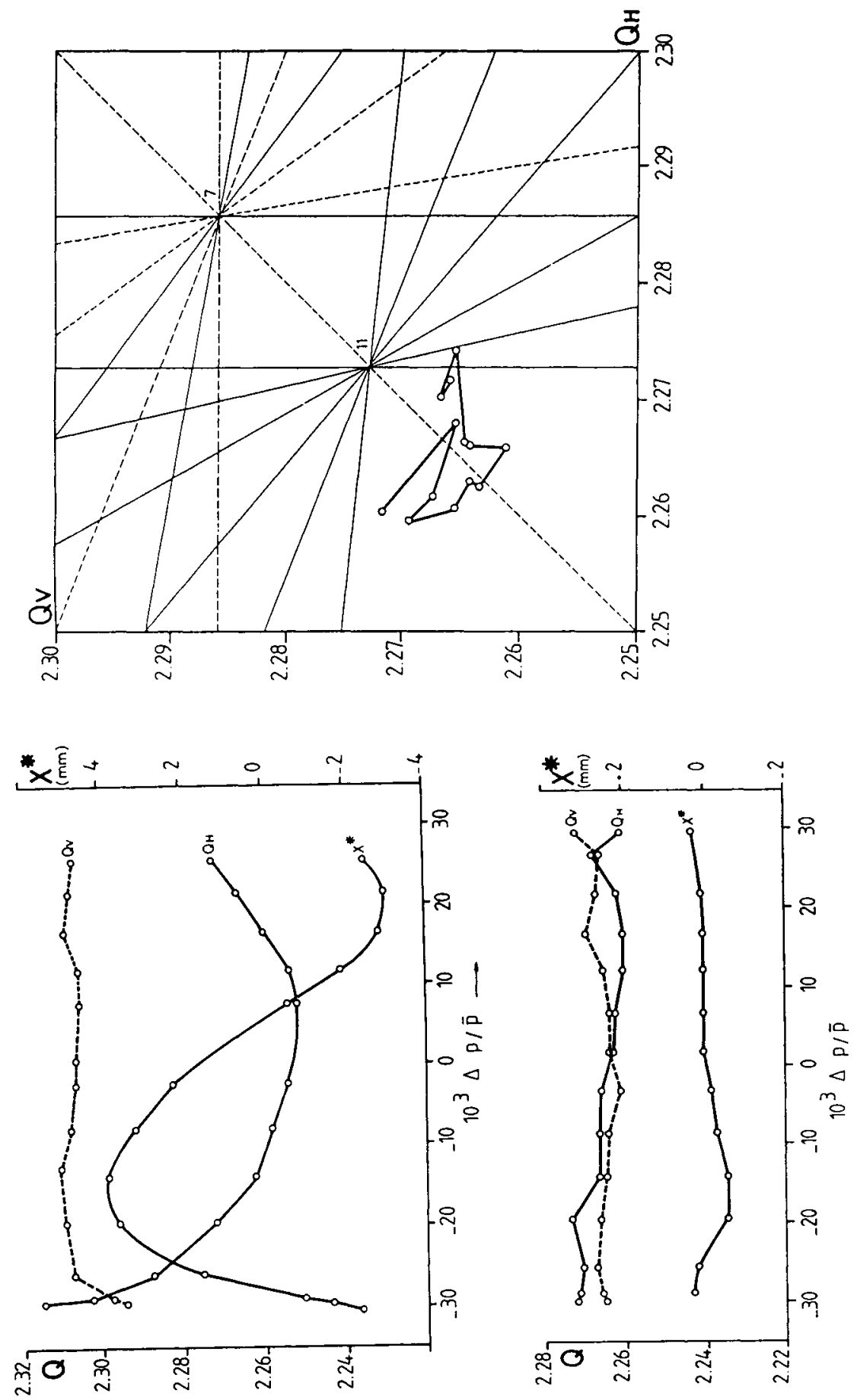

$\stackrel{2}{a}$ 


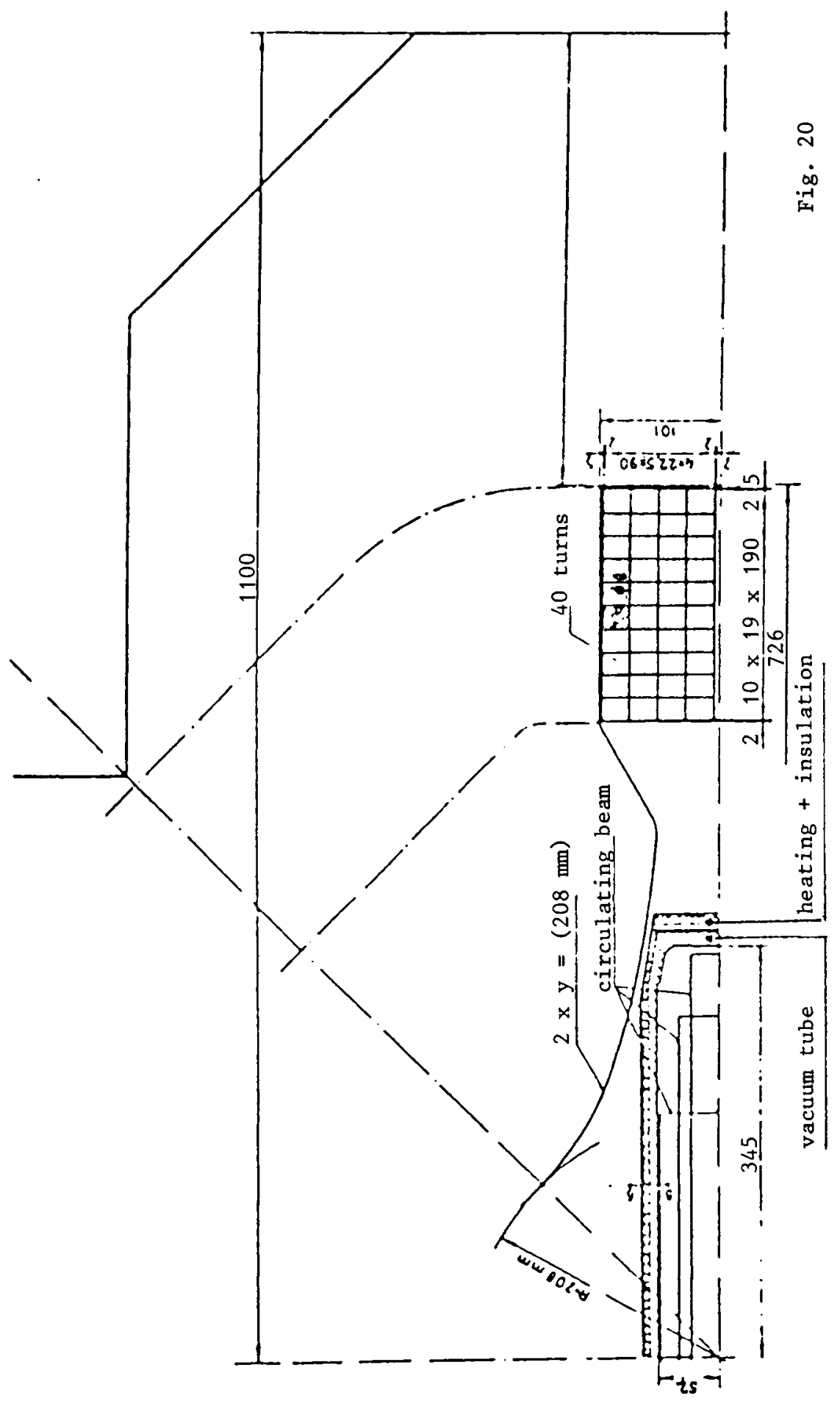




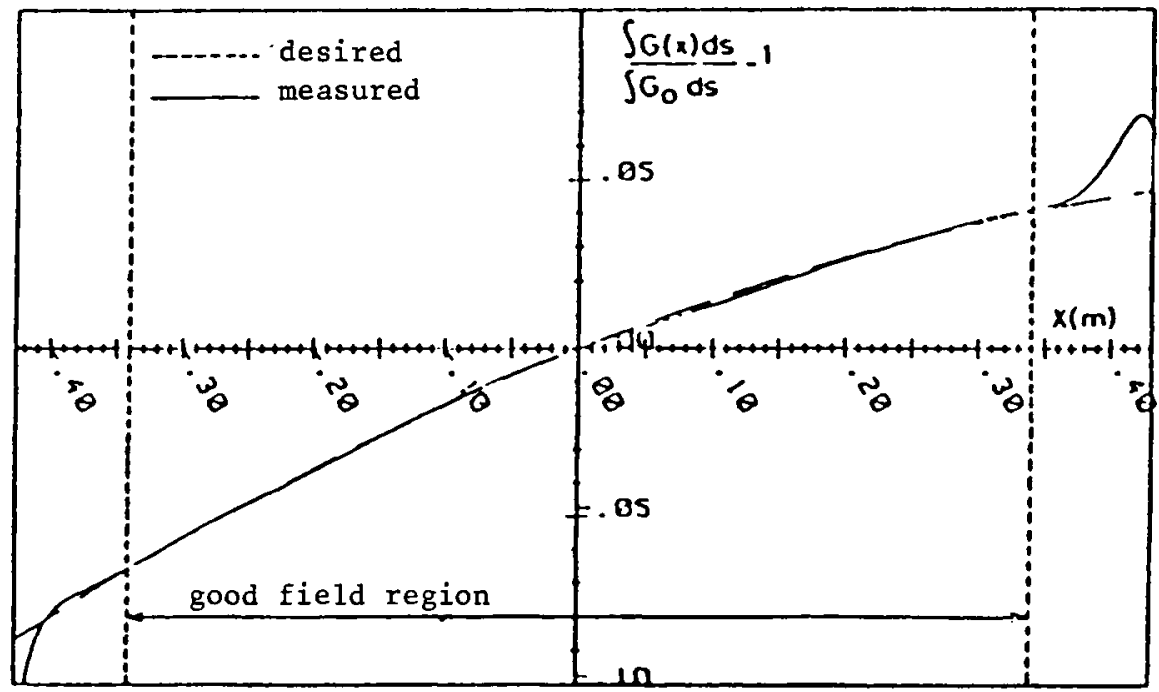

Fig. 21

cooling. The precooling is designed to drastically reduce the momentum spread of the injected pulse by an order of magnitude so that a rather weak RF system can capture, decelerate, and deposit it gently in the tail of the stack in time for a new pulse to take its place. The size of the RF bucket $V=(\Delta p / p)^{2}$ is just proportional to the momentum spread after precooling. If it is too big the bucket will disturb the tail of the stack sufficiently to phase displace particles back outwards and hit the shutters as they close again.

Once in the tail, the stack tail cooling system takes over. It finds the particles having a lower frequency than the stack because their momentum and mean radius are different and it applies a signal to a cooling kicker which decelerates them towards the stack frequency. On their way their horizontal and vertical betatron amplitudes are reduced by stack tail transverse systems.

Once in the stack, a third set of cooling systens take over. These are the high frequency core cooling systems designed for intense beams. We find in Ref. 4 that it is a characteristic of cooling systems that they are most rapid in their effect on small groups of particles.

In the same reference is to be found an expression for the rate of cooling:

$$
\frac{1}{t}=\frac{2 W}{N}\left[2 g-g^{2}(1+0)\right] \approx \frac{2 W}{N}
$$

where $g$ is the gain $\left(g_{\text {opt }}\right)=1 /(1+e)$,

$p$ is the noise/signal power,

$W$ is the bandwidth,

$\mathrm{N}$ is the number of particles. 


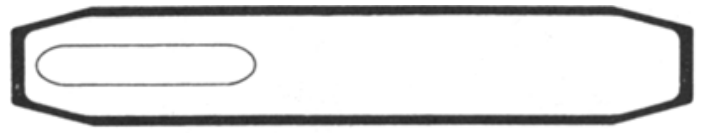

The first pulse is injected into the vacuum chamber.

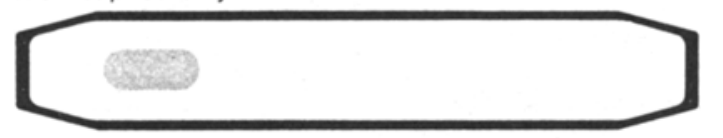

Precooling is applied.

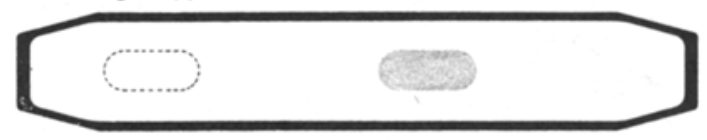

The pulse is moved into the stack position.

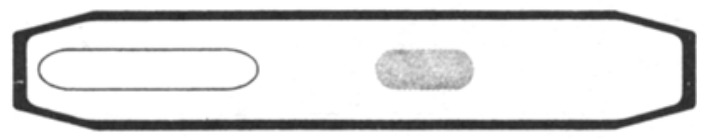

The second pulse is injected 2.2 seconds later.

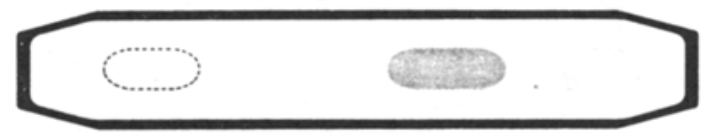

The second pulse is stacked after being precooled.

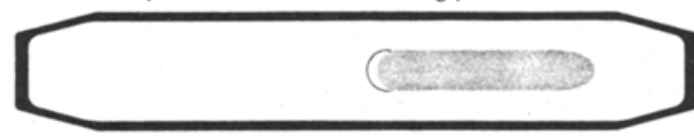

150 pulses later, the stack intensity is $10^{\circ}$ antiprotons.

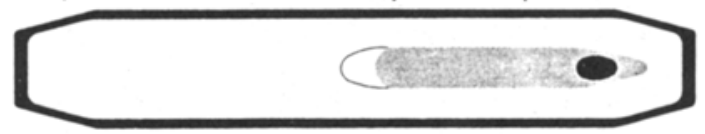

After 3 hours, a dense core is forming in the stack.

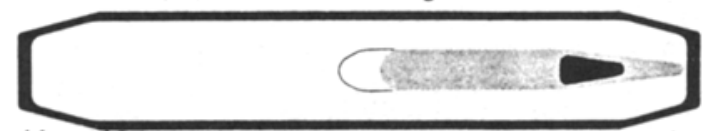

After 120 hours the core contains enough antiprotons to be ejected.

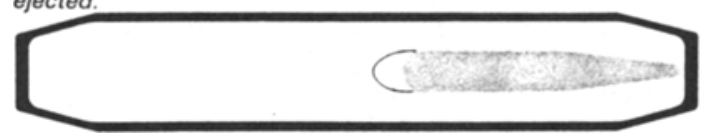

The remaining antiprotons are used to start the next core.

Fig. 22 
The few million pbars in each injected pulse can be momentum cooled by the precooling system in a matter of 1 or 2 seconds, but it takes much longer for the core cooling systems to shrink the emittance of a stack containing 1011 or more antiprotons. They must detect and correct stochastic fluctuations of a much larger population which are a much smaller fraction of the mean signal.

It takes many hours for the core to build up to the density needed by the SPS. Once it does and the beam is ripe for transfer, a small bucket is formed by the RF at a frequency within a bell-shaped spectrum of stack frequencies. The bunch of particles it catches is accelerated past the open shutters to the old injection orbit where the ejection kicker fires to make it jump the septum.

\section{THE COOLING SYSTEMS}

transverse

pick-up

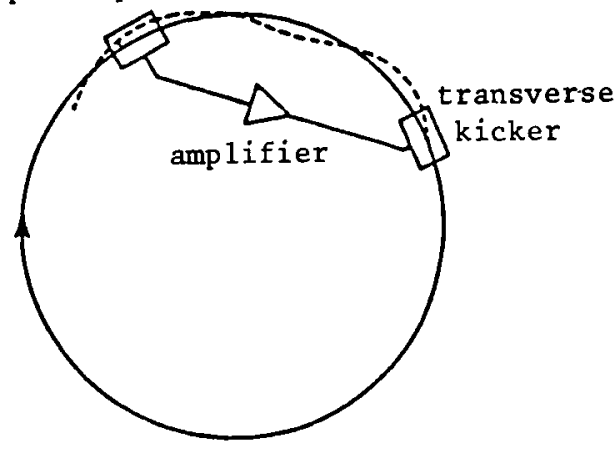

The precooling system 13 is a straightforward application of the principle of stochastic momentum cooling. The aim is to accelerate particles with too low a momentum and decelerate those with too high a momentum. A full description of cooling is to be found elsewhere. Here we shall merely recall those qualitative concepts necessary to understand the rest of this paper. Suppose a rogue particle, one with too high a momentum, passes a pick-up along with a large number of other particles (Figs. 23 and Fig. 23 $24 a)$. The rogue shifts the centre of gravity of the horizontal charge distribution (Fig. 24b) in its direction and tells the pick-up to send a message across the ring so that it arrives, amplified, at an accelerating kicker just when the rogue does. The kicker attempts to correct the aberrant tendency of the rogue by applying an accelerating or decelerating voltage (Fig. 24c). If there is more than one rogue with the same tendency so much the better, if there are two rogues with equal and opposite tendencies nothing happens on that turn. But the two rogues will not remain together because of the natural mixing due to their different frequencies and sooner or later they will be singled out to be dealt with individually (Fig. 24d).

Gradually, turn after turn, the rogue always gets a kick in the right direction. It sees a coherent signal which cools it.

of course, all other well-behaved particles tend to get heated incoherently as they become associated with different rogues in the mixing process, but this incoherent effect is proportional to the square of the gain of the kicker amplifier, while cooling is linearly proportional to the gain. There is therefore a region of gain adjustment where there is a net cooling for the beam as a whole. 

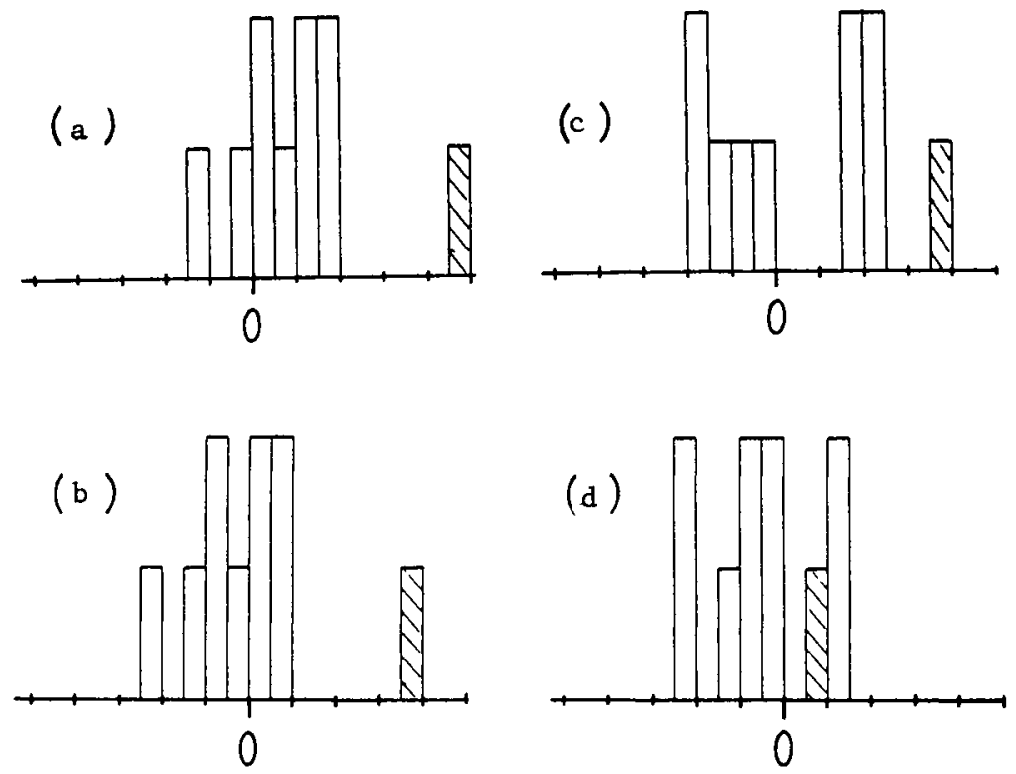

Fig. 24

PRECOOLING SYSTEM

The way in which the rogue particles are detected varies for the different cooling systems. The precooling system discriminates between particles of different frequencies by filters which give zero response at the revolution frequency (and all its harmonics) of the central momentum. They give a positive signal for too high a momentum and negative signal for too low a momentum.

One can calculate the rate of cooling for a given bandwidth and number of particles from Equation 16. For the precooling system this must reduce $\delta \mathrm{p} / \mathrm{p}$ from \pm 0.75 to $1 / 8$ th of this in 2 seconds (Fig. 25). This is possible, but represents quite a large transfer of energy to

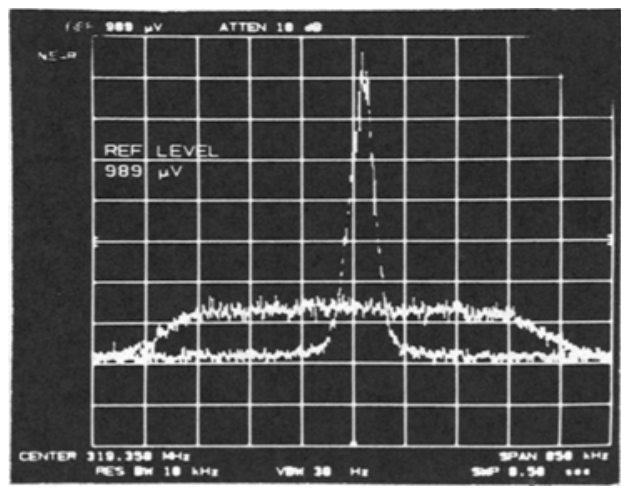

Fig. 25 
the beam so that the electromagnetic coupling to the beam must be tight. This is achieved by making the precooling elements, kicker and pick-up, in the form of ferrite rings enclosing the beam (Fig. 26). They form a fast transformer excited as a kicker, by a primary winding with the beam as its secondary. The transformer has a mechanical shutter on one side, where a slab of ferrite, part of the yoke, can be raised to protect the stack against stray field and lowered to let the fresh pulse join the stack.

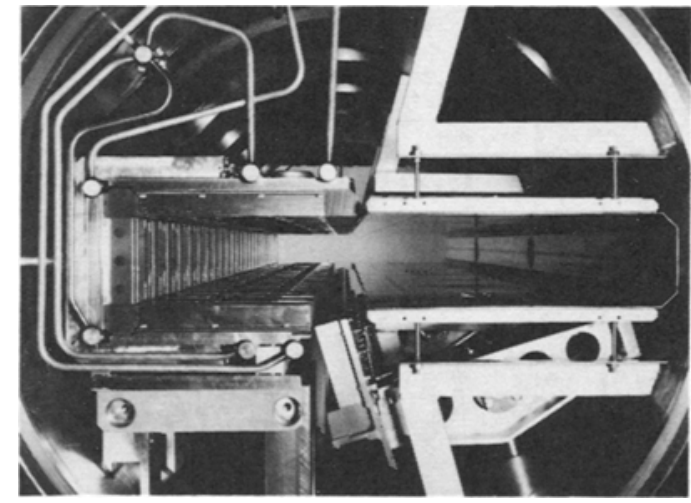

Fig. 26

Such a system cannot respond to a very high frequency since the ferrite will absorb power above $300 \mathrm{MHz}$ and become inefficient.

\section{THE RF SYSTEM}

Although modest in voltage the $R F$ is required to perform a number of functions. The cavity is of conventional design. A dual-gap ferrite-loaded structure, driven by a 4CX 25000 tetrode, can be tuned over the range of frequencies from 1844 to $1856 \mathrm{kMHz}$ which correspond to revolution frequencies of pbars at the edges of the vacuum chamber. Figure 27 shows its low level system. The voltage and frequency may be controlled manually to adiabatically capture a test beam of protons and move it across the vacuum chamber to measure closed orbit position, Q, dispersion, or acceptance.

During normal pulsed accumulation there is a sequence of manoeuvres in frequency and voltage set up by an interactive program.14 The RF is off during injection and precooling, but, once this is complete, the voltage rises in $200 \mathrm{~ms}$ to adiabatically capture the beam. With voltage and frequency programmed to keep the area of the moving bucket constant, the antiprotons are decelerated past the shutters, which are opened in synchronism. The bucket is then made to shrink as it is brought to a halt at a frequency in the tail of the stack, depositing its burden as it collapses. The whole sequence takes 400 ms from start to finish. 


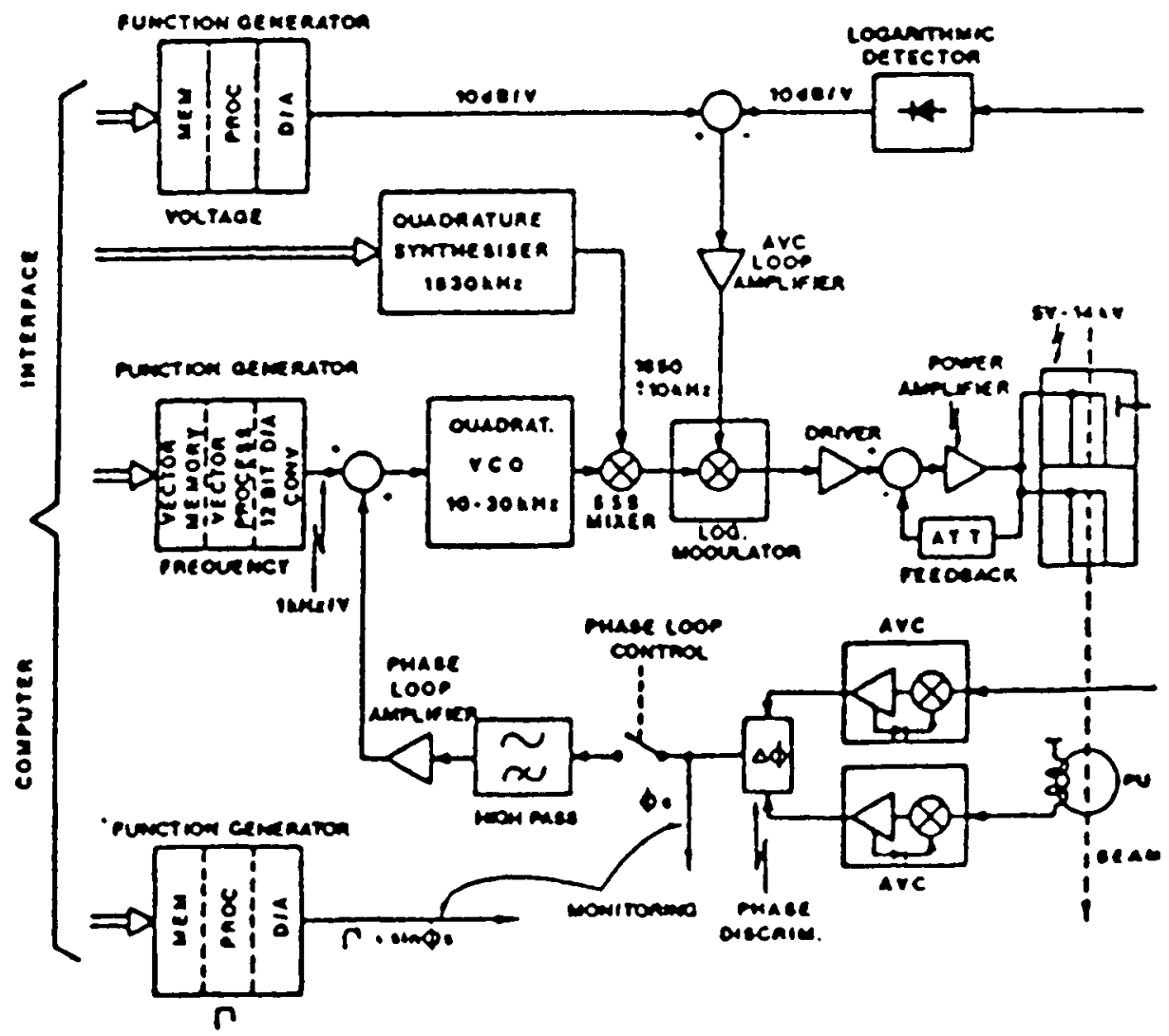

Fig. 27

The process of capture from the stack is very similar except that here the bucket needs to be very small and the RF voltage as low as 5 volts. Special phase-control and beam-loading feedback loops allow this. By using a spectrum analyzer to measure the stack density distribution, the capture frequency and voltage can be chosen to take either a small percentage of the stack for test purposes or as much as 158 for each of the 3 bunches needed to fill the SPS.

\section{THE STACK TAIL COOLING SYSTEM}

This cooling system merges the deposited bunch with the stack. It must cover a wide range of spectral density from the tenuous stack tail to the edge of the dense core. Its gain must be carefully profiled as a function of frequency so that the few particles just deposited in the tail are displaced rapidly away before the next pulse, while higher up the flank of the stack, where there are more particles, the response is attenuated to avoid excessive power levels. Ideally the profile of gain should promote a progressively slower 
diffusion rate which results in the sort of stack tail shape shown in the computer prediction (Fig. 28). The variation in density spans 4 orders of magnitude.

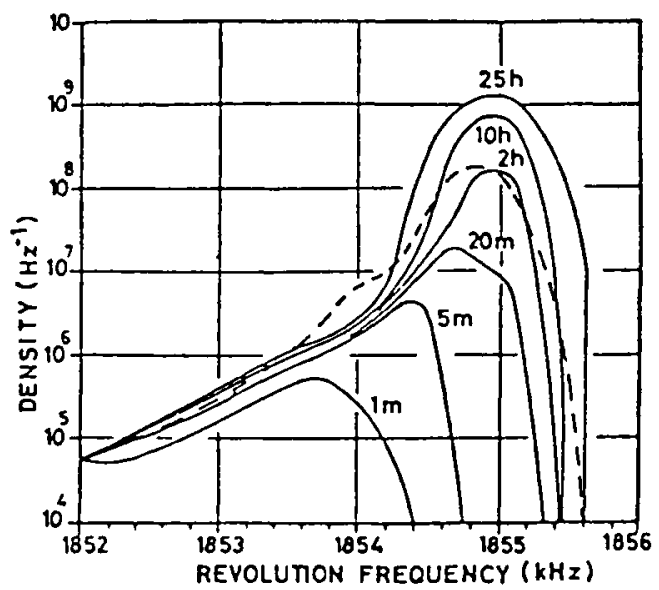

Fig. 28

To achieve this there are really three different pick-up systems with different sensitivities placed at different radial positions in the arcs of the AA where there is dispersion ( $90^{\prime}$ clock in Fig. 2 ). Their filters are tuned to progressively higher frequencies so that they pass on particles in the direction of the stack.

The noise from the stack tail system can be rather large, especially from the amplifiers dealing with the pick-ups. There is a danger that it may cause heating. The noise can be considerably reduced by refrigerating the load resistor and preamplifier of the pick-up and this is done.

\section{STACK CORE COOLING}

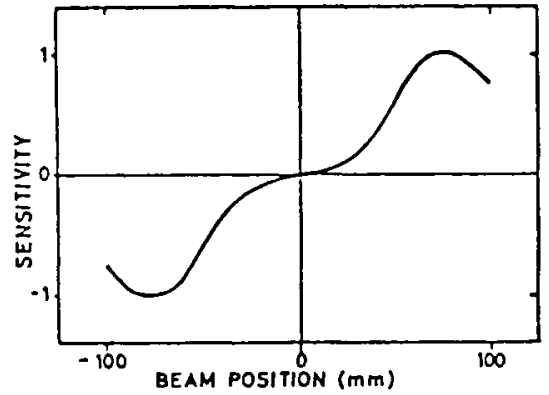

Fig. 29

The stack-core cooling system has a hard job which it can only do slowly. Its time constant is of the order of half an hour. To cool a stack of 1011 or more antiprotons, the highest possible bandwidth, $W$, is used. Power and coupling to the beam on the other hand are not at a premium. It is logical on both accounts to use high frequencies. Since most broad-band systems can be made to cover an octave, the absolute bandwidth increases with frequency. Unlike the precooling system whose pick-ups have ferrite rings and where filters provide the error signal, the core system pick-ups produce a signal which is position sensitive, the palmer method (Fig. 29). 
Such pick-ups are slotted transmission lines working in the 1 - to $2-\mathrm{GHz}$ region. They have two rows of slots; top and bottom. Difference signals can be used to pick up betatron oscillations, and sum signals detect momentum changes.

Kickers have the configuration shown in Fig. 30. Correction of betatron motion is fed in in push-pull mode and correction of momentum deviation in common mode.

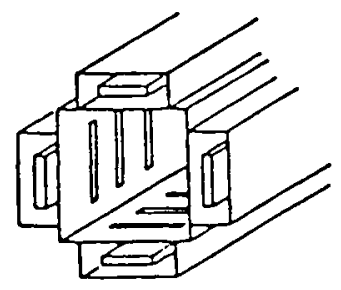

Fig. 30

\section{PERFORMANCE AND IMPROVEMENTS}

Although the AA works well and stacks of $3 \times 1011 \bar{p}$ are commonplace, the discovery of $W$ 's and $Z^{\prime}$ 's has whetted the appetite for more detailed physics demanding higher spps luminosities. This places heavier demands not only on the SPS but on the AA.

A number of factors act together to limit further improvements of the AA. The most fundamental of these is its acceptance. Ways have been found to build conducting targets and lithium-lens collection systems which collect four times the flux from the target by increasing the acceptance angle. Spectrometers can be designed which bring four times the energy spread to the AA, but with this 16-fold increase in flux there is inevitably a comparable increase in phase space. The beam would be too big for the AA.

One could imagine redesigning the $A A$ with a tighter lattice (more quadrupoles) whose acceptance per centimetre of magnet aperture would then be larger. However, this would destroy the carefully chosen compromise between acceptance and the dispersion necessary to isolate injected beam and stack. Even if this could be resolved, the present AA cooling systems would need to be replaced with higher frequency systems whose pick-ups in the 4- to 8-GHz range would have to be much smaller than the present beam dimensions.

The only solution is to cut the Gordian knot and build a special collector ring around the $A A$, the $A C$, with a tighter lattice and bigger phase space acceptance and then perform momentum and transverse cooling in this ring to squeeze the beam before entering the AA (Fig. 31). The AA would then be modified to have high frequency systems which could handle the higher flux. The new ring will essentially take over the precooling function of the outer part of the AA and pass on its beam each pulse to the $A A$.

Freed from the constraints of having two beams in one machine, other possibilities open up. The AC or precooling ring can use bunch rotation preceding stochastic cooling and almost instantaneously reduce the momentum spread of the incoming antiprotons. This bunch rotation, as will be used at FNAL, exploits use of the fact that the incoming bunches have a mark-space ratio of about 1:5. Injected into a stationary RF bucket the tall, short bunch rotates in a quarter synchrotron oscillation, becoming long and shallow in momentum (Fig. 32). 


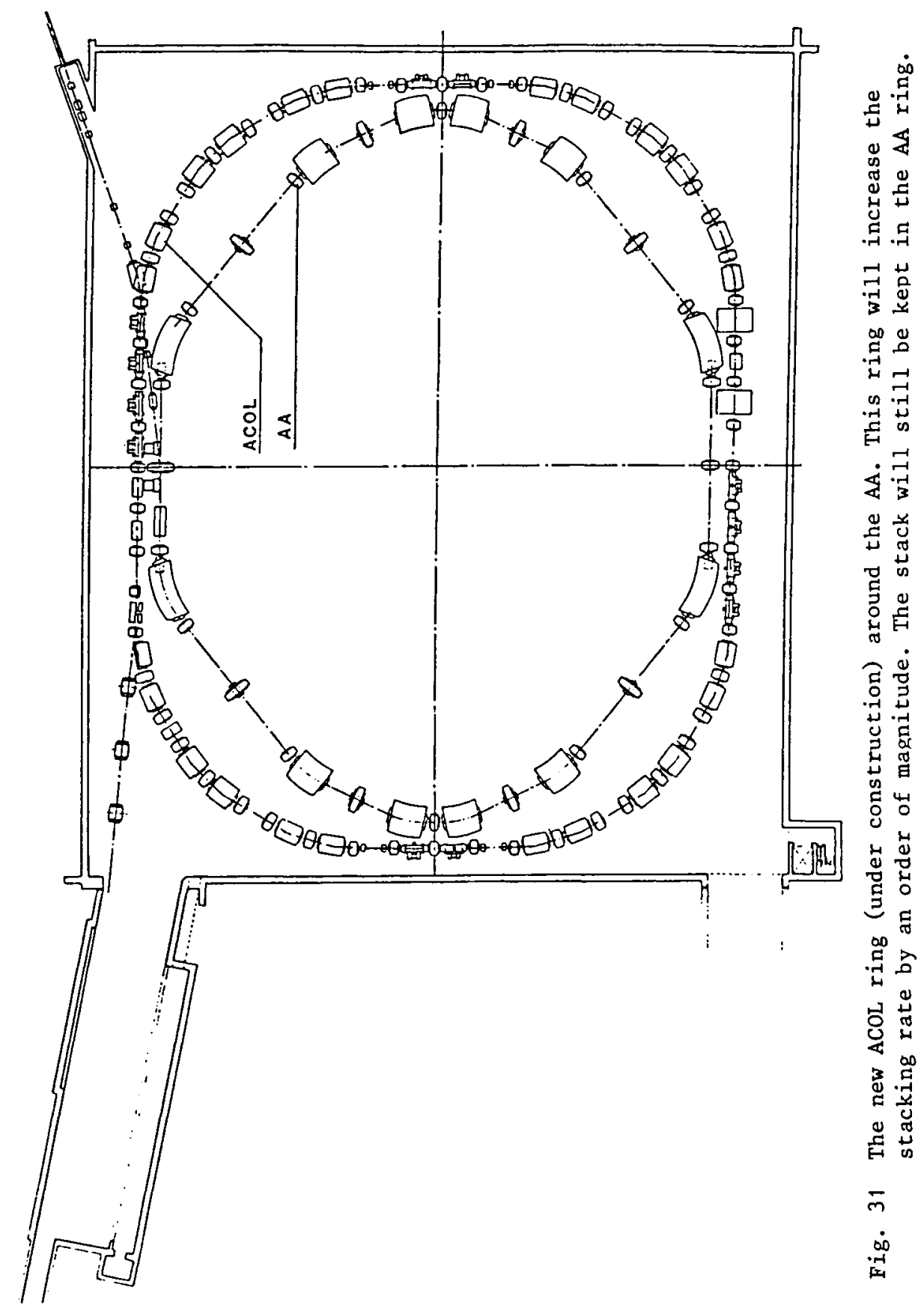




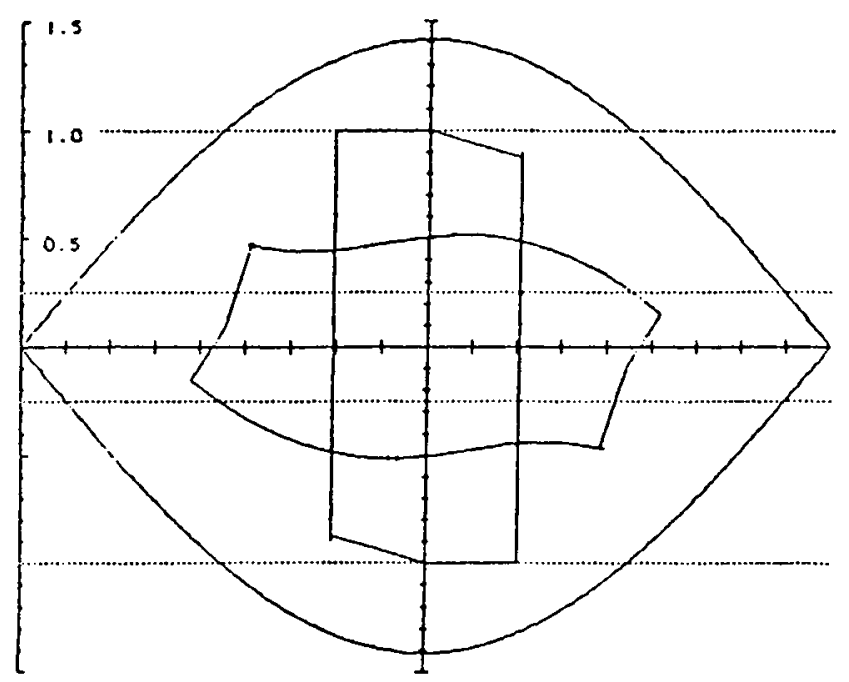

Fig. 32

The betatron precooling necessary in the AC is possible with present-day technology within the 2 seconds available because of new solid-state broad-band amplifiers working in the 2- to 4-GHz region. The pick-ups are rather inefficient if they are made large enough to accept the injected beam but we can squeeze them down with mechanical pistons to become more efficient as the beam shrinks.

All these tricks are embodied in the antiproton collector project, ACOL, now underway at CERN. The new ring, concentric with the $A A$, is due to work in 1987 providing an order of magnitude improvement in antiproton flux. Meanwhile of course our colleagues at FNAL are advancing in their own antiproton source which has many of the features of CERN's ACOL.

\section{REFERENCES}

1. L. Evans, This volume.

2. D. Boussard, This volume.

3. R. Billinge, This volume.

4. S. van der Meer, This volume.

5. R. Bellone et al., Proc. Workshop on High Temp. Energy Density in Target Materials, FNAL (1980).

6. D. Dekkers et al., Phy. Rev. 137 B, 962 (1965).

7. C. Hojvat and A. van Ginneken, Nucl. Instrum. Methods 206 Nos. 1,2 (1983).

8. J.V. Allaby, Antiproton Production (1984), Unnumbered CERN/EP Internal Report.

9. E. Jones, $\bar{p}$ production and Collection, CERN/PS-AA/83-46 (1983).

10. B. Autin, Proc. Part. Acc. Conf., San Francisco (1979).

11. R. Brown et al., Proc. 7th Int. Conf. on Magnet Technology, Karlsruhe (1981).

12. G. Gelato et al., Proc. Part. Acc. Conf., Washington (1981).

13. S. van der Meer, Proc. Part. Acc. Conf., Washington (1981).

14. R. Johnson et al., Proc. Part. Acc. Conf., Santa Fe (1983). 